ESAIM: PROCEEDINGS, December 2012, Vol. 38, p. 1-35

F. Coquel, M. Gutnic, P. Helluy, F. Lagoutière, C. Rohde, N. Seguin, Editors

\title{
TWO-SCALE CONVERGENCE
}

\author{
EMMANUEL FRÉNOD ${ }^{1}$
}

\begin{abstract}
Those notes are the lecture notes of lectures given at Cemracs 2011 Summer School. I present here the classical results of Two-Scale Convergence Theory and an application to Homogenization of linear Singularly Perturbed Hyperbolic Partial Differential Equations.

Résumé. Ces notes constituent les notes du cours donné à l'école d'été du Cemracs 2011. Je présente les résultats classiques de la théorie de la convergence à deux échelles et une application à l'homogénéisation d'équations aux dérivées partielles hyperboliques linéaires singulièrement perturbées.
\end{abstract}

\section{Contents}

1. Introduction 2

1.1. On Two-Scale Convergence first statements 2

1.2. How Homogenization brought the concept 2

$\begin{array}{ll}\text { 1.3. A remark concerning periodicity } & 10\end{array}$

1.4. A remark concerning weak-* convergence 10

2. Two-Scale Convergence - Definition and Results 14

$\begin{array}{ll}\text { 2.1. Definitions } & 14\end{array}$

\begin{tabular}{llr}
\hline 2.2. & Link with Weak Convergence & 15
\end{tabular}

$\begin{array}{llr}2.3 . & \text { Injection Lemma } & 16\end{array}$

2.4. Two-Scale Convergence criterion $\quad 19$

$\begin{array}{lll}2.5 . & \text { Strong Two-Scale Convergence criterion } & 21\end{array}$

3. Application : Homogenization of linear Singularly Perturbed Hyperbolic Equations 23

$\begin{array}{lll}3.1 . & \text { Equation of interest and setting } & 23\end{array}$

$\begin{array}{lll}\text { 3.2. A priori estimate } & 24\end{array}$

3.3. Weak Formulation with Oscillating Test Functions 24

3.4. Order 0 Homogenization - Constraint 24

3.5. Order 0 Homogenization - Equation for $V \quad 25$

3.6. Order 1 Homogenization - Preparations: equation for $U$ and $u$.

3.7. Order 1 Homogenization - Strong Two-Scale convergence of $U$

3.8. Order 1 Homogenization - Function $W_{1} \quad 28$

3.9. Order 1 Homogenization - A priori estimate and convergence 29

3.10. Order 1 Homogenization - Constraint 30

3.11. Order 1 Homogenization - Equation for $V_{1}$

${ }^{1}$ Université Européenne de Bretagne, Lab-STICC (UMR CNRS 3192), Université de Bretagne-Sud, Centre Yves Coppens, Campus de Tohannic, F-56017, Vannes \& Projet INRIA Calvi, Université de Strasbourg, IRMA, 7 rue René Descartes, F-67084 Strasbourg Cedex, France

(c) EDP Sciences, SMAI 2012 


\section{INTRODUCTION}

\subsection{On Two-Scale Convergence first statements}

The concept of Two-Scale Convergence was introduced in two papers of Nguetseng [27, 28 in 1989. Then in 1992, Allaire 3] produced a synthetic and very readable proof of the result.

\subsection{How Homogenization brought the concept}

The concept of Two-Scale Convergence emerged from questions of Periodic Homogenization. Homogenization is a Mathematical Theory, or more precisely, an Asymptotic Analysis Theory that originates from Material Engineering, or more precisely, from understanding the way Constitutive Equation of composite material can be gotten from Constitutive Equation of each component of the concerned material and from their topological and geometrical distributions.

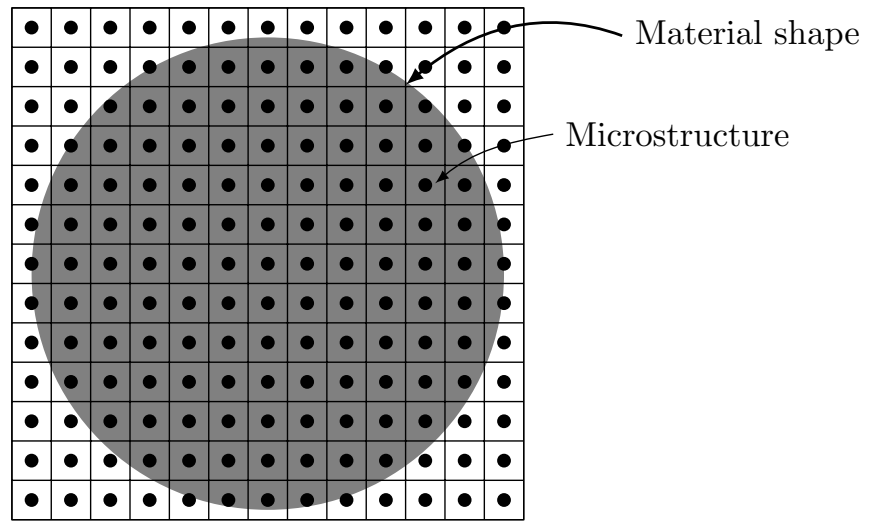

Figure 1.1. Composite material has a macroscopic shape and a microstructure. The ratio between the size of the microstructure and the size of the material is $\varepsilon$.

In order to make the purpose clear, we first consider the simplest - but rich enough - example I know. (The explanation that follows does not aim to be mathematically rigorous. It clearly appeals to intuition and to non-rigorous vocabulary.)

Imagine that we want to get the temperature field within a composite material which is in thermal equilibrium, knowing the temperature on its boundary. Symbolically, as represented in Figure 1.1 (in a bi-dimensional setting), the composite material has a macroscopic shape at a macroscopic size. Within it, heterogeneities are more or less periodically distributed with a periodicity - or a characteristic size - which is $\varepsilon$ times smaller than its macroscopic size, where $\varepsilon$ is a small parameter. This makes up what is usually called the microstructure of the composite material. Now, to achieve our goal, we contemplate the following Heat Equation

$$
\begin{aligned}
& \nabla \cdot\left[a^{\varepsilon}\left(\mathbf{x}, \frac{\mathbf{x}}{\varepsilon}\right) \nabla u^{\varepsilon}\right]=0 \quad \text { within the material, } \\
& u^{\varepsilon} \text { given on the boundary of the material, }
\end{aligned}
$$


that is supposed to describe how the temperature $u^{\varepsilon}$ is being split within the material from its distribution on the boundary. In this equation, $a^{\varepsilon}$ stands for the Thermal Diffusion Coefficient (it is the ratio Thermal Conduction over Calorific Capacity times material Density), $\nabla$ and $\nabla \cdot$ stand for the gradient and divergence operators. (If a unidimensional material is considered, $\mathbf{x}=x$ lives in $\mathbb{R}$, if a bi-dimensional material is considered, $\mathbf{x}=(x, y)$ lives in $\mathbb{R}^{2}$ and if a tridimensional material is considered, $\mathbf{x}=(x, y, z)$ lives in $\mathbb{R}^{3}$.)

The fact that $a^{\varepsilon}$ depends on $\mathbf{x}$ and $\mathbf{x} / \varepsilon$ needs to be understood in the following sense. Variable $\mathbf{x}$ is the dimensionless position, meaning that when used to describe the material at its macroscopic scale, the needed variations of variable $\mathbf{x}$ are of the order of 1 . Beside this, the dependence of $a^{\varepsilon}$ in $\mathbf{x} / \varepsilon$ models the variation of the Thermal Diffusion at the microstructure scale. To illustrate this ability of $\mathbf{x} / \varepsilon$-dependence to describe variations at the microscopic scale, I show the graph of some kinds of functions in one and two dimensions. In Figure 1.2 , is drawn the graph, between $-\pi$ and
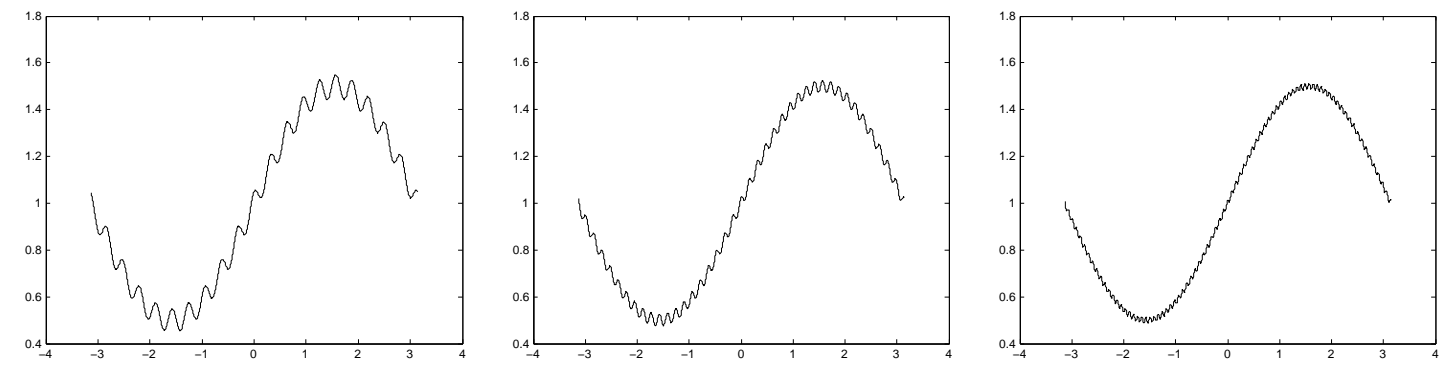

Figure 1.2. Graph of $\frac{1}{2} \sin (x)+1+\varepsilon \cos \left(\frac{x}{\varepsilon}\right)$ for $\varepsilon=1 / 20$ (left), $1 / 40$ (center) and $1 / 80$ (right) between $-\pi$ and $\pi$.

$\pi$, of $a^{\varepsilon}(x, x / \varepsilon)=(1 / 2) \sin (x)+1+\varepsilon \cos (x / \varepsilon)$ for $\varepsilon=1 / 20,1 / 40$ and $1 / 80$. Those functions have a variation at the macroscopic scale, which is described by the piece $(1 / 2) \sin (x)+1$ and variations at much smaller scales, which are the microscopic variations. In this example, the microscopic variations can be qualified of being high frequency periodic oscillations with small amplitude. They are described by the term $\cos (x / \varepsilon)$ which needs to be multiplied by $\varepsilon$ (explaining the presence of superscript $\varepsilon$ in $a^{\varepsilon}$ ) to insure amplitude of size $\varepsilon$ of those high frequency periodic oscillations. The
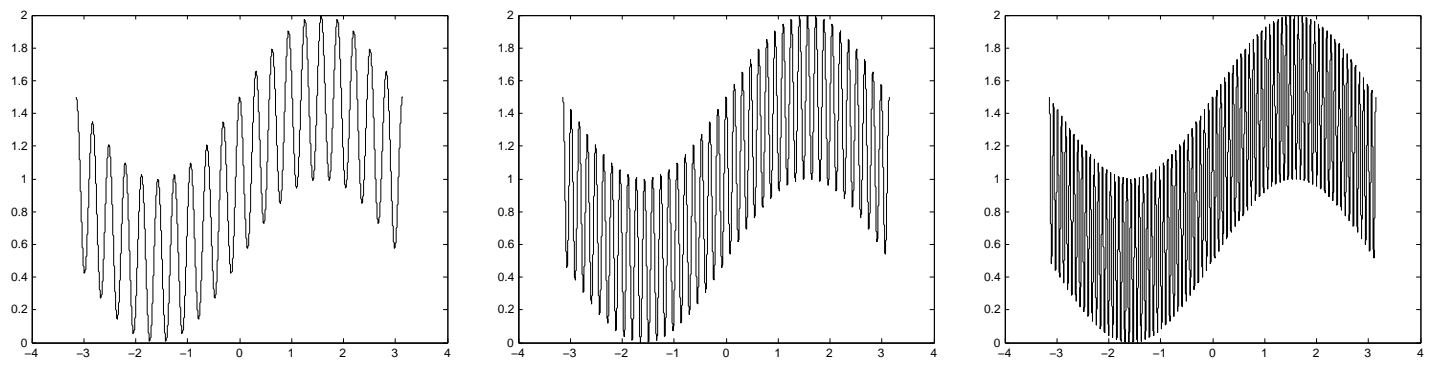

Figure 1.3. Graph of $\frac{1}{2} \sin (x)+1+\frac{1}{2} \cos \left(\frac{x}{\varepsilon}\right)$ for $\varepsilon=1 / 20$ (left), $1 / 40$ (center) and $1 / 80$ (right) between $-\pi$ and $\pi$.

next figure (figure 1.3) shows the graph of $a^{\varepsilon}(x, x / \varepsilon)=a(x, x / \varepsilon)=(1 / 2) \sin (x)+1+(1 / 2) \cos (x / \varepsilon)$ for the same values of $\varepsilon$ as previously. Here, the macroscopic scale variation is always given by the 
piece $(1 / 2) \sin (x)+1$ and the variations at a smaller scale, making up the microscopic variations, are given by $(1 / 2) \cos (x / \varepsilon)$. (This term is not multiplied by $\varepsilon$, bringing the uselessness of superscript $\varepsilon$ in $a^{\varepsilon}$.) In this case the microscopic variations can be qualified of high frequency periodic oscillations with large amplitude or periodic Strong Oscillations. Figure 1.4 shows the ability of a function
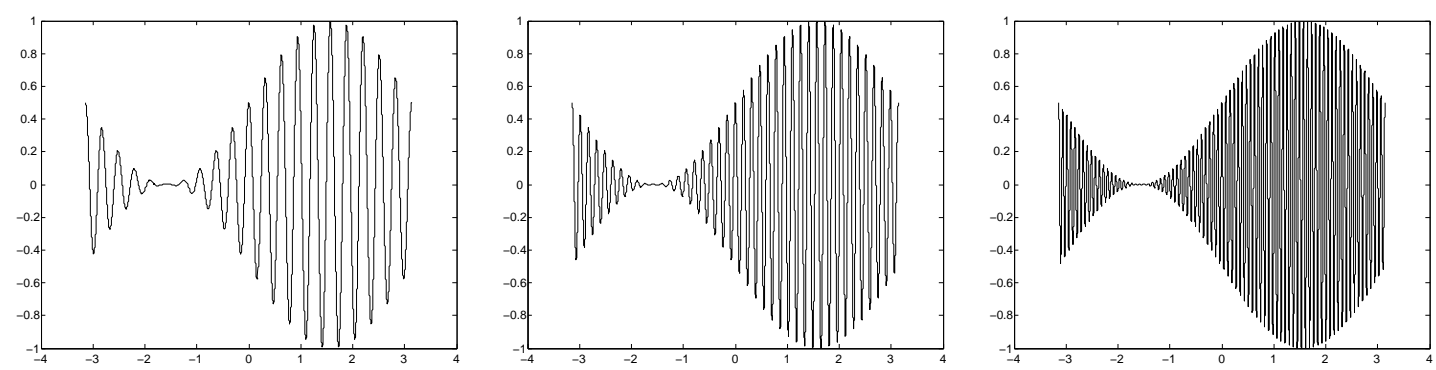

FIGURE 1.4. Graph of $\frac{1}{2}(\sin (x)+1) \cos \left(\frac{x}{\varepsilon}\right)$ for $\varepsilon=1 / 20$ (left), $1 / 40$ (center) and $1 / 80$ (right) between $-\pi$ and $\pi$.

depending on $x$ and $x / \varepsilon$ to describe situations with microscopic variations which are with modulated amplitude - with regions where Oscillations are Strong and regions where they are not Strong. The drawn function is $(1 / 2)(\sin (x)+1) \cos (x / \varepsilon)$. In this expression, the microscopic variations, which are high frequency periodic oscillations, are described by factor $\cos (x / \varepsilon)$ and the modulated amplitude is $(1 / 2)(\sin (x)+1)$. Figure 1.5 shows function $(1 / 2) \cos (x)+1+(1 / 2)(\sin (x)+1) \cos (x / \varepsilon)$ for always
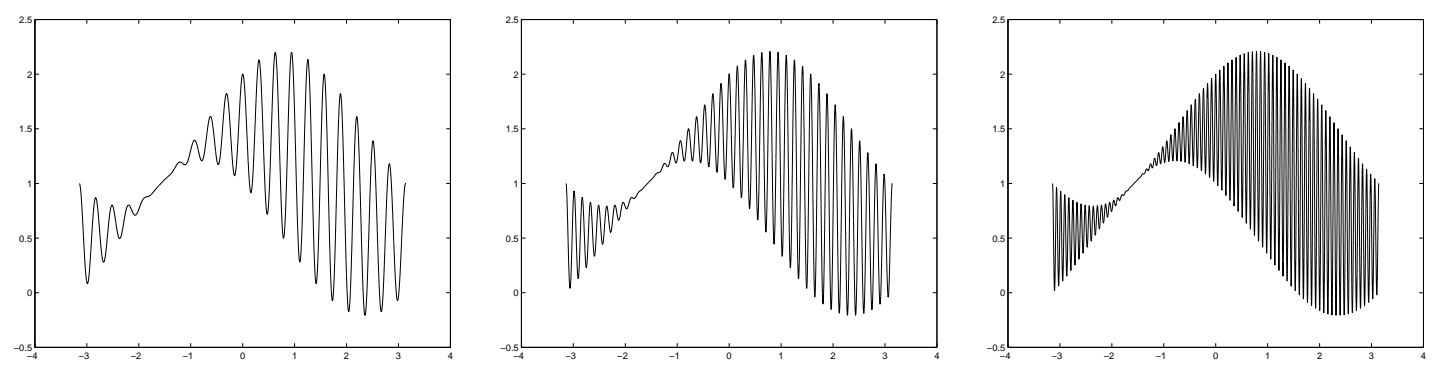

FIGURE 1.5. Graph of $\frac{1}{2} \cos (x)+1+\frac{1}{2}(\sin (x)+1) \cos \left(\frac{x}{\varepsilon}\right)$ for $\varepsilon=1 / 20$ (left), $1 / 40$ (center) and $1 / 80$ (right) between $-\pi$ and $\pi$.

the same values of $\varepsilon$. Those functions possesses both macroscopic scale variation and modulated amplitude high frequency oscillations as microscopic variations.

In every example above the microscopic scale variations are periodic. Yet, the $x / \varepsilon$-dependence may also describe microscopic scale variations that are not periodic. This is illustrated in figure 1.6 where function $(1 / 10) \sin (x / 3)+1+(1 / 4) \cos (x / \varepsilon) \sin ((\pi / 4) x / \varepsilon)$ is drawn.

Despite the fact that, for visibility reasons, the chosen value of $\varepsilon$ is not very small $(1 / 20)$, figures 1.7 and 1.8 show bi-dimensional functions with periodic Strong Oscillations (figure 1.7) and modulated amplitude in one direction (figure 1.8).

At the end of the day, functions writing $a^{\varepsilon}(x, x / \varepsilon)$ have the ability to describe a variety of coupling both macroscopic and microscopic variations which is wide enough. 

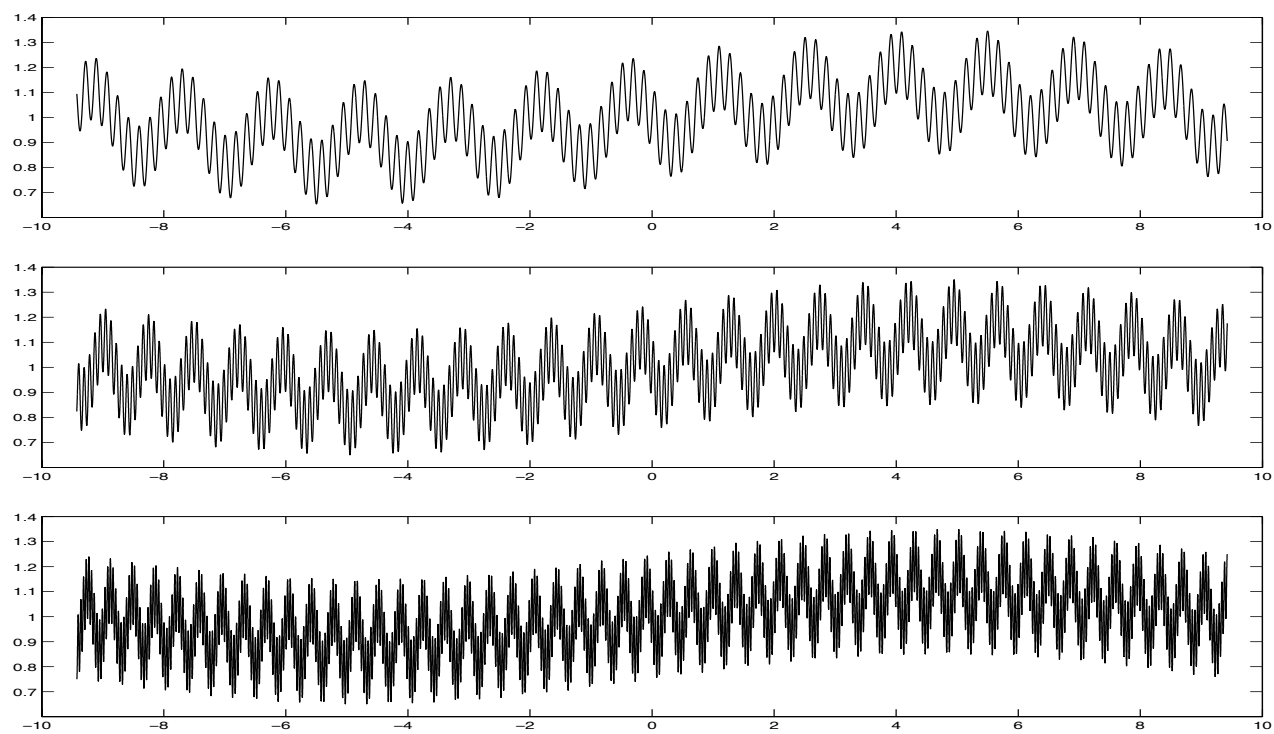

FiguRE 1.6. Graph of $\frac{1}{10} \sin \left(\frac{x}{3}\right)+1+\frac{1}{4} \cos \left(\frac{x}{\varepsilon}\right) \sin \left(\frac{\pi}{4} \frac{x}{\varepsilon}\right)$ for $\varepsilon=1 / 20$ (top), $1 / 40$ (center) and $1 / 80$ (bottom) between $-3 \pi$ and $3 \pi$.
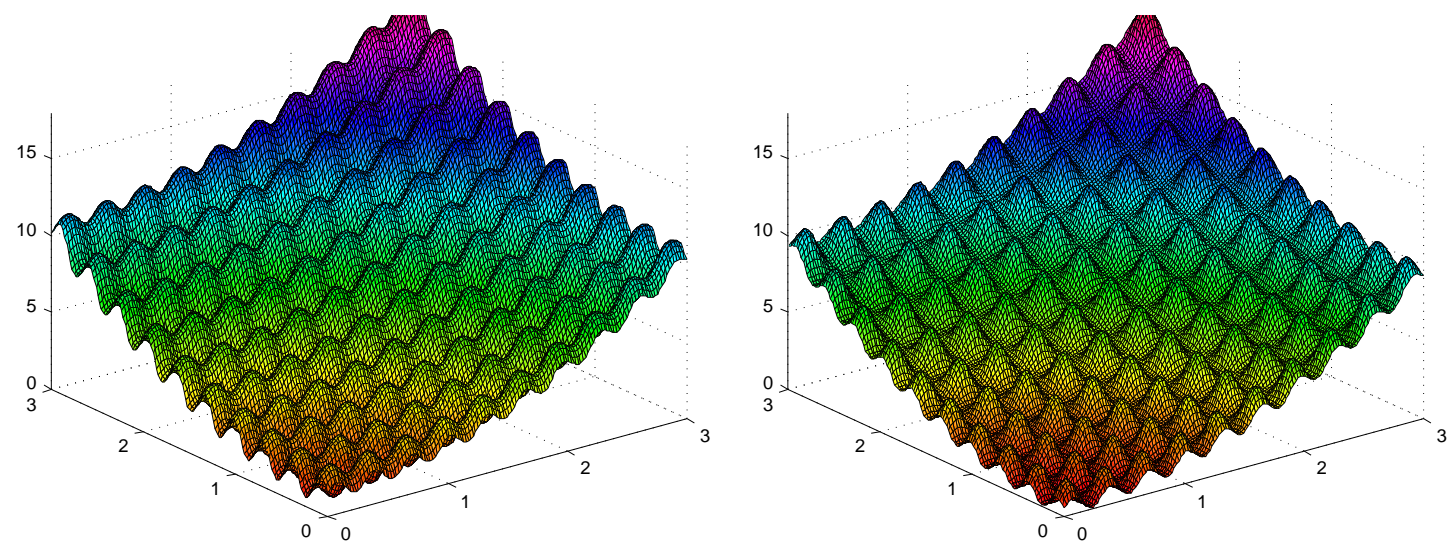

Figure 1.7. Graph of $x^{2}+y^{2}+\frac{1}{2}\left(\sin \left(\frac{y}{\varepsilon}\right)+1\right)+\left(\sin \left(\frac{x}{\varepsilon}\right)+1\right)$ (left) and of $x^{2}+y^{2}+$ $\frac{1}{2}\left(\sin \left(\frac{y}{\varepsilon}\right)+1\right)\left(\sin \left(\frac{x}{\varepsilon}\right)+1\right)$ (right) for $\varepsilon=1 / 20$ on $[0,3]^{2}$.

It is practicable to introduce a variable (say $\boldsymbol{\xi}$, which may be $\xi,(\xi, v)$ or $(\xi, v, \zeta)$, depending on the dimension number) which describes the variations at the microscopic scale. This consists in considering that $a^{\varepsilon}$ in fact depends on two variables: $a^{\varepsilon}(\mathbf{x}, \boldsymbol{\xi})$ and that the coefficient in equation (1.1) is

$$
a^{\varepsilon}\left(\mathbf{x}, \boldsymbol{\xi}=\frac{\mathbf{x}}{\varepsilon}\right) .
$$




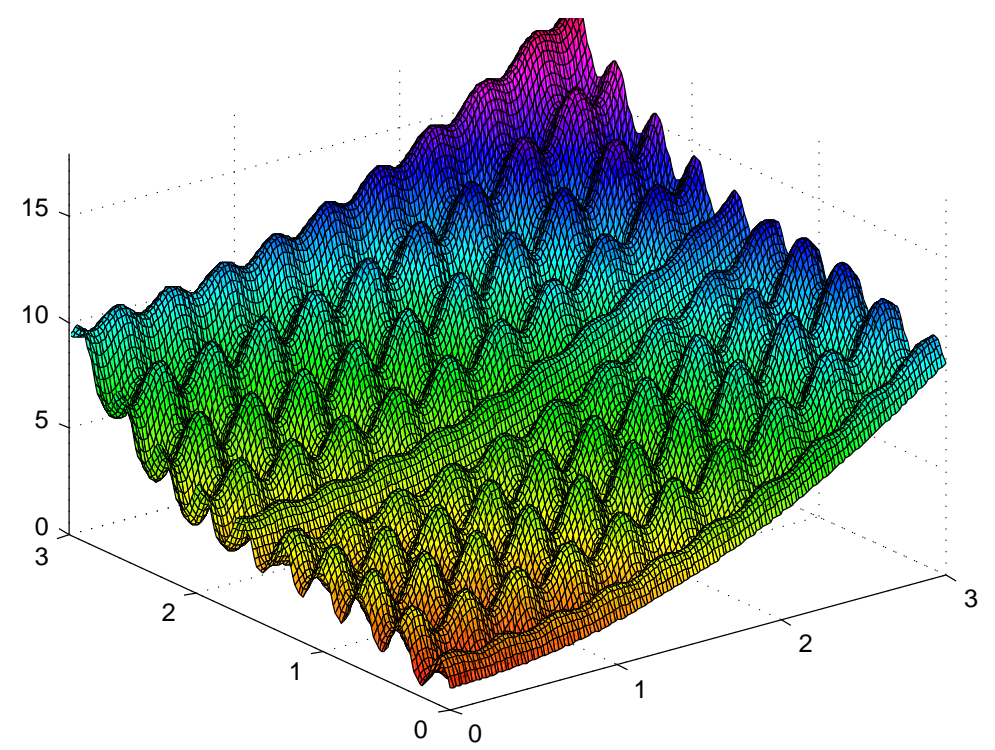

Figure 1.8. Graph of $x^{2}+y^{2}+\sin (2 x)\left(\sin \left(\frac{y}{\varepsilon}\right)+1\right)+\left(\sin \left(\frac{x}{\varepsilon}\right)+1\right)$ for $\varepsilon=1 / 20$ on $[0,3]^{2}$.

Applying this practicable trick to the examples involved in figures above, the following formulas are gotten:

$$
\begin{aligned}
& a^{\varepsilon}(x, \xi)=\frac{1}{2} \sin (x)+1+\varepsilon \cos (\xi), \text { in the case of figure 1.2. } \\
& a^{\varepsilon}(x, \xi)=a(x, \xi)=\frac{1}{2} \sin (x)+1+\frac{1}{2} \cos (\xi), \text { in the case of figure 1.3, } \\
& a^{\varepsilon}(x, \xi)=a(x, \xi)=\frac{1}{2}(\sin (x)+1) \cos (\xi), \text { in the case of figure 1.4. } \\
& a^{\varepsilon}(x, \xi)=a(x, \xi)=\frac{1}{2} \cos (x)+1+\frac{1}{2}(\sin (x)+1) \cos (\xi), \text { in the case of figure 1.5. } \\
& a^{\varepsilon}(x, \xi)=a(x, \xi)=\frac{1}{10} \sin \left(\frac{x}{3}\right)+1+\frac{1}{4} \cos (\xi) \sin \left(\frac{\pi}{4} \xi\right), \\
& a^{\varepsilon}(x, y, \xi, v)=a(x, y, \xi, v)=x^{2}+y^{2}+\frac{1}{2}(\sin (v+1)+(\sin (\xi)+1), \text { and } \\
& a^{\varepsilon}(x, y, \xi, v)=a(x, y, \xi, v)=x^{2}+y^{2}+\frac{1}{2}(\sin (v)+1)(\sin (\xi)+1) \\
& \text { in the case of figure } 1.7 \text { and, } \\
& a^{\varepsilon}(x, y, \xi, v)=a(x, y, \xi, v)=x^{2}+y^{2}+\frac{1}{2}(\sin (v)+1)(\sin (\xi)+1), \text { in the case of figure } 1.8 .
\end{aligned}
$$

If $\boldsymbol{\xi} \mapsto a^{\varepsilon}(\mathbf{x}, \boldsymbol{\xi})$ is periodic, the microscopic scale variations are qualified of high frequency periodic oscillations.

Remark 1.1. Two-Scale Convergence is essentially designed to be used in the context of high frequency periodic oscillations.

Going back to the question we imagine we are interested in, we need to implement a numerical method, for instance a Finite Difference Method or a Finite Element Method, in order to compute 
an approximated solution of Partial Differential Equation 1.1. Since, $\mathbf{x}$ is a dimensionless variable, the domain on which (1.1) is set has a size which order of magnitude is 1 . But, to get a reasonable result, we must choose a discretization step $\Delta x$ which is such that $\Delta x<<\varepsilon$. Otherwise, the effect of the microstructure is not taken into account, and the resulting computation has nothing to do with reality. Hence, if $\varepsilon$ is very small, meaning that the microstructure is much smaller than the macroscopic size, the computation can be very expensive and even not feasible. For instance, if we consider a tridimensional material, with $\varepsilon=10^{-3}$ then, with the constraint $\Delta x<<\varepsilon$, that we consider to be achieved with $\Delta x=10^{-1} \varepsilon$, the order of magnitude of the number of degrees of freedom needed for the computation is $\left(10 \cdot 10^{3}\right)^{3}=10^{12}$. This is quite expensive. Leading such a computation may not be completely unreasonable if we are interested in knowing the intimate distribution of the temperature at the microstructure scale, but in most of the situations the tiny variations at this scale have no interest. In those cases, it is highly unreasonable to lead such a heavy computation to get the result.

Hence, we would like to have on hand an equation, which does not explicitly involve any microstructure, but which contains, or more precisely, which induces in its solution the average effect of the microstructure (which is described by the $\mathbf{x} / \varepsilon$-dependence of $a^{\varepsilon}$ in 1.1). Denoting symbolically this equation by

$$
\mathcal{H} u=0
$$

involving an operator $\mathcal{H}$, with the constraint that $u$ is close to $u^{\varepsilon}$ in some senses, we can expect to implement a numerical method to compute an approximated solution of $(1.4)$ - which is also an approximation of $u^{\varepsilon}$ - with a cost much smaller (because the constraint $\Delta x<<\varepsilon$ is useless) than the one needed for the direct approximation of equation 1.1 .

Homogenization Theory gathers a collection of methods that allows us to build operators $\mathcal{H}$ satisfying the required constraint.

The first Homogenization methods were set out by Engineers in the middle of the 1970s and then formalized by Mechanical Scientists. They are based on Asymptotic Expansion. Applying them in the case of equation (1.1) consists in writing

$$
u^{\varepsilon}(\mathbf{x})=U\left(\mathbf{x}, \frac{\mathbf{x}}{\varepsilon}\right)+\varepsilon U_{1}\left(\mathbf{x}, \frac{\mathbf{x}}{\varepsilon}\right)+\varepsilon^{2} U_{2}\left(\mathbf{x}, \frac{\mathbf{x}}{\varepsilon}\right)+\ldots,
$$

with functions $U(\mathbf{x}, \boldsymbol{\xi}), U_{1}(\mathbf{x}, \boldsymbol{\xi}), U_{2}(\mathbf{x}, \boldsymbol{\xi}), \ldots$ periodic with respect to $\boldsymbol{\xi}$, in injecting this expansion in equation 1.1), in identifying and gathering terms in factor of $\varepsilon^{-2}, \varepsilon^{-1}, \varepsilon^{0}, \varepsilon, \varepsilon^{2}, \ldots$ and then in deducing a set of equations:

$$
\begin{aligned}
& H_{-2} U=0, \\
& H_{-1} U_{1}=\mathcal{I}(U), \\
& H_{0} U_{2}=\mathcal{I}^{\prime}\left(U, U_{1}\right), \\
& \ldots
\end{aligned}
$$

Extracting information from those equations, we get well-posed equations for $U, U_{1}, U_{2}, \ldots$

For a full understanding of the methods based on Asymptotic Expansion, I refer to the books by Sanchez-Palencia 33 and to the one by Bensoussan, Lions \& Papanicolaou [5]. 
Then, if we want to get a rigorous mathematical justification of the process just described, we need to prove results like

$$
\left\|u^{\varepsilon}(\mathbf{x})-U\left(\mathbf{x}, \frac{\mathbf{x}}{\varepsilon}\right)\right\|_{?} \rightarrow 0
$$

for a norm \|\|$_{\text {? }}$ to be determined, or in a weaker sense,

$$
\left(u^{\varepsilon}(\mathbf{x})-U\left(\mathbf{x}, \frac{\mathbf{x}}{\varepsilon}\right)\right) \rightarrow 0 .
$$

If we want to get justifications at higher orders, we need to prove convergence results like

$$
\left(\frac{u^{\varepsilon}(\mathbf{x})-U\left(\mathbf{x}, \frac{\mathbf{x}}{\varepsilon}\right)}{\varepsilon}-U_{1}\left(\mathbf{x}, \frac{\mathbf{x}}{\varepsilon}\right)\right) \rightarrow 0
$$

in some senses,

$$
\left(\frac{1}{\varepsilon}\left(\frac{1}{\varepsilon}\left(u^{\varepsilon}(\mathbf{x})-U\left(\mathbf{x}, \frac{\mathbf{x}}{\varepsilon}\right)\right)-U_{1}\left(\mathbf{x}, \frac{\mathbf{x}}{\varepsilon}\right)\right)-U_{2}\left(\mathbf{x}, \frac{\mathbf{x}}{\varepsilon}\right)\right) \rightarrow 0,
$$

in some senses, and so on.

In the case of a Parabolic Partial Differential Equation like (1.1), with Dirichlet boundary conditions, convergence results of the kind of $(1.9)$ can be brought out using the Maximum Principle and boundary estimates (see Bensoussan, Lions \& Papanicolaou [5]).

In other cases, the way is less straightforward, and appeals to Oscillating Test Functions used within a Weak Formulation of the Partial Differential Equation. Passing to the limit using Compensated Compactness like results (see Tartar 36 ) may give convergence results of the kind of (1.9) or (1.10). This method is called "Energy Method" or "Oscillating Test Function Method" and was designed by Tartar 35] in collaboration with Murat [26 (see also [5]). For mathematical justification, works of Engquist have also to be consulted, in particular 11 .

The Weak Formulation with Oscillating Test Functions writes, in the case of equation (1.1),

$$
\int_{\text {Material }} \nabla \cdot\left[a^{\varepsilon}\left(\mathbf{x}, \frac{\mathbf{x}}{\varepsilon}\right) \nabla u^{\varepsilon}(\mathbf{x})\right] \varphi\left(\mathbf{x}, \frac{\mathbf{x}}{\varepsilon}\right) d \mathbf{x}=0,
$$

which yields, using the Stokes Formula,

$$
\int_{\text {Material }} a^{\varepsilon}\left(\mathbf{x}, \frac{\mathbf{x}}{\varepsilon}\right) \nabla u^{\varepsilon}(\mathbf{x}) \cdot \nabla\left[\varphi\left(\mathbf{x}, \frac{\mathbf{x}}{\varepsilon}\right)\right] d \mathbf{x}=\int_{\text {Boundary }} \text { Something, }
$$

or

$$
\int_{\text {Material }} a^{\varepsilon}\left(\mathbf{x}, \frac{\mathbf{x}}{\varepsilon}\right) \nabla u^{\varepsilon}(\mathbf{x}) \cdot\left[\nabla_{\mathbf{x}} \varphi\left(\mathbf{x}, \frac{\mathbf{x}}{\varepsilon}\right)+\frac{1}{\varepsilon} \nabla_{\boldsymbol{\xi}} \varphi\left(\mathbf{x}, \frac{\mathbf{x}}{\varepsilon}\right)\right] d \mathbf{x}=\int_{\text {Boundary }} \text { Something. }
$$

In those integrals, $\nabla u^{\varepsilon}$ converges generally in a weak sense only and this is the same for the other involved functions: $a^{\varepsilon}\left(\mathbf{x}, \frac{\mathbf{x}}{\varepsilon}\right), \nabla_{\mathbf{x}} \varphi\left(\mathbf{x}, \frac{\mathbf{x}}{\varepsilon}\right)$ and $\nabla_{\boldsymbol{\xi}} \varphi\left(\mathbf{x}, \frac{\mathbf{x}}{\varepsilon}\right)$. It is well known that passing to the limit in a product of two weak converging sequences of functions is a non-straightforward task. Hence, passing to the limit in 1.13, 1.14 or 1.15 is not so easy and consequently involves relatively sophisticated analytical methods (like Compensated Compactness results).

The situation just described is typical of the mathematical justification of homogenization results. 
Two-Scale Convergence offers an efficient framework to pass to the limit in such terms, in the case when oscillations are periodic. It is certainly possible to infer that Two-Scale Convergence emerges from those kind of questioning. Yet, as it will be illustrated by the example treated in section 3. Two-Scale Convergence is much more than a method to justify Asymptotic Expansion: it is a constructive Homogenization Method very well adapted to Singularly Perturbed Hyperbolic Equations.

Two-Scale Convergence is a homogenization tool which is well adapted to situations involving periodicity. In the non periodic setting more refined tools are needed. I refer to the book of Tar$\operatorname{tar} 38$ for the descriptions of those methods. Among them, $G$-convergence (see Pankov 32]) is well adapted to problem involving Green Kernel, $\Gamma$-convergence (see Dal Maso [9] and Braides [7]) is a tool designed for homogenization of optimization problems. I also mention the very sophisticated $H$-measures of Tartar [37] and Gérard [24 that allow to tackle fine homogenization questions within the micro-local calculus framework.

Of course, we can try to provide an answer to questions of the same type as before in other field than composite material. For instance we can be interested in the time varying version of [1.1]:

$$
\begin{aligned}
& \frac{\partial u^{\varepsilon}}{\partial t}-\nabla \cdot\left[a^{\varepsilon}\left(\mathbf{x}, \frac{\mathbf{x}}{\varepsilon}\right) \nabla u^{\varepsilon}\right]=0 \quad \text { within the material, } \\
& u^{\varepsilon}(t, .) \text { given on the boundary of the material, } \\
& u^{\varepsilon}(0, .) \quad \text { given at initial time within the material, }
\end{aligned}
$$

where $t$ stands for a dimensionless time and where $\mathbf{x}$ has the same meaning as in equation 1.1 .

We can also consider the following equations :

$$
\frac{\partial z^{\varepsilon}}{\partial t}-\frac{1}{\varepsilon} \nabla \cdot\left[a\left(t, \frac{t}{\varepsilon}, \mathbf{x}\right) \nabla z^{\epsilon}\right]=\frac{1}{\varepsilon} \nabla \cdot \mathbf{c}\left(t, \frac{t}{\varepsilon}, \mathbf{x}\right),
$$

and

$$
\frac{\partial z^{\varepsilon}}{\partial t}-\frac{1}{\varepsilon^{2}} \nabla \cdot\left[a\left(t, \frac{t}{\varepsilon}, \mathbf{x}\right) \nabla z^{\epsilon}\right]=\frac{1}{\varepsilon^{2}} \nabla \cdot \mathbf{c}\left(t, \frac{t}{\varepsilon}, \mathbf{x}\right),
$$

which are relevant models for the short-term and long-term dynamics of dunes on a seabed of a coastal ocean where tide is strong. In equation (1.17) and (1.18), $z^{\varepsilon}=z^{\varepsilon}(t, \mathbf{x})$, where $t$ stands is the dimensionless time and $\mathbf{x}$ is the bi-dimensional dimensionless position variable, is the dimensionless seabed altitude at time $t$ and in position $\mathbf{x}$. Those equations were widely studied in Faye, Frénod \& Seck 12,13 .

We can also be interested in the following Vlasov equations :

$$
\frac{\partial f^{\varepsilon}}{\partial t}+\mathbf{v} \cdot \nabla_{\mathbf{x}} f^{\varepsilon}+\left(\mathbf{E}(\mathbf{x}, t)+\frac{1}{\varepsilon} \mathbf{v} \times \mathbf{B}(\mathbf{x}, t)\right) \cdot \nabla_{\mathbf{v}} f^{\varepsilon}=0,
$$

and

$$
\frac{\partial f^{\varepsilon}}{\partial t}+\mathbf{v}_{\|} \cdot \nabla_{\mathbf{x}} f^{\varepsilon}+\frac{1}{\varepsilon} \mathbf{v}_{\perp} \cdot \nabla_{\mathbf{x}} f^{\varepsilon}+\left(\mathbf{E}(\mathbf{x}, t)+\frac{1}{\varepsilon} \mathbf{v} \times \mathbf{B}(\mathbf{x}, t)\right) \cdot \nabla_{\mathbf{v}} f^{\varepsilon}=0
$$

which are models involved in Tokamak Plasma physics. In those equations $\mathbf{x} \in \mathbb{R}^{3}$ stands for the dimensionless position, $\mathbf{v} \in \mathbb{R}^{3}$ for the dimensionless velocity and $t$ for the dimensionless time. The solution $f^{\varepsilon}=f^{\varepsilon}(t, \mathbf{x}, \mathbf{v})$ which is also dimensionless is, at time $t$ the density of ions in position $\mathbf{x}$ and with velocity $\mathbf{v}$. Field $\mathbf{E}$ is the Electric field and field $(1 / \varepsilon) \mathbf{B}(\mathbf{x}, t)$ is the Strong Magnetic field. We denote by $\|$ and $\perp$ the directions parallel and perpendicular to this magnetic field. 
Those two last equations involve neither oscillating coefficients nor any microstructure. Nevertheless, the strong magnetic field induces in the solution high frequency periodic oscillations. Equations 1.19 or 1.20 can be cast into the following framework of a singularly perturbed convection equation:

$$
\frac{\partial u^{\varepsilon}}{\partial t}+\mathbf{a} \cdot \nabla u^{\varepsilon}+\frac{1}{\varepsilon} \mathbf{b} \cdot \nabla u^{\varepsilon}=0, \quad \mathbf{x} \in \mathbb{R}^{d}, t>0,
$$

by setting, in the case of 1.19 ,

$$
\mathbf{a}(\mathbf{x}, \mathbf{v}, t)=\left(\begin{array}{c}
\mathbf{v} \\
\mathbf{E}(t, \mathbf{x})
\end{array}\right) \text { and } \mathbf{b}(\mathbf{x}, \mathbf{v})=\left(\begin{array}{c}
0 \\
\mathbf{v} \times \mathbf{B}(t, \mathbf{x})
\end{array}\right),
$$

and, in the case of 1.20 ,

$$
\mathbf{a}(\mathbf{x}, \mathbf{v}, t)=\left(\begin{array}{c}
\mathbf{v}_{\|} \\
\mathbf{E}(t, \mathbf{x})
\end{array}\right) \text { and } \mathbf{b}(\mathbf{x}, \mathbf{v})=\left(\begin{array}{c}
\mathbf{v}_{\perp} \\
\mathbf{v} \times \mathbf{B}(t, \mathbf{x})
\end{array}\right)
$$

Equation of this type are studied in Frénod \& Sonnendrücker 20 22, Frénod \& Watbled 23, Frénod, Raviart \& Sonnendrücker [18], Frénod 14]15], Frénod \& Hamdache [16 Ailliot, Frénod \& Monbet 1, 2], Frénod, Mouton \& Sonnendrücker [17] and Frénod, Salvarani \& Sonnendrücker [19] .

\subsection{A remark concerning periodicity}

Two-Scale Convergence is well-adapted to the framework of high frequency periodic oscillations (or to cases that can be brought to this framework by an adequate transformation). But, it essentially does not work in non-periodic cases. Even in the case of oscillations with a period depending on the variable describing the macroscopic variation, it does not work. Many questions linked with non-periodic homogenization are essentially open.

\subsection{A remark concerning weak-* convergence}

Here, I give the proof of two important and representative results. They concern the characterization of the weak limit of functions with high frequency periodic oscillations.

I will denote, for $p=1, \ldots, \infty$, by $L^{p}(\mathbb{R})$ the space of functions defined almost everywhere on $\mathbb{R}$ such that their $p$-th power is Lebesgue integrable, by $L_{\#}^{p}(\mathbb{R})$ the space of functions being in $L^{p}(\mathbb{R})$ and periodic of period 1 , by $\mathcal{C}_{\#}^{0}(\mathbb{R})$ the space of functions being continuous over $\mathbb{R}$ and periodic of period 1 , by $\mathcal{C}^{\infty}(\mathbb{R})$ the space of functions being infinitely derivable over $\mathbb{R}$ and by $\mathcal{D}(\mathbb{R})$ the space of functions being in $\mathcal{C}^{\infty}(\mathbb{R})$ and compactly supported. Finally, we denote by $L^{p}\left(\mathbb{R} ; \mathcal{C}_{\#}^{0}(\mathbb{R})\right)$ the Lebesgue space of Bochner integrable functions mapping $\mathbb{R}$ to $\mathcal{C}_{\#}^{0}(\mathbb{R})$. This space can be characterized as the space of functions mapping $\mathbb{R}$ to $\mathcal{C}_{\#}^{0}(\mathbb{R})$ such that the $p$-th power of their norm is Lebesgue integrable if $p<\infty$ and such that their norm is essentially bounded if $p=\infty$. (I refer the reader to Bochner [6], Diestel \& Uhl [10, Yosida 39] and Schwartz 34] for a precise and thoroughly description of Integration Theory, specifically for functions with values in Banach spaces.)

The first result, which is often referred to as the "Riemann-Lebesgue Lemma", gives the asymptotic behavior, with respect to weak topology, of a periodic function applied in $\xi=x / \varepsilon$.

Lemma 1.2. Let $\psi \in L_{\#}^{\infty}(\mathbb{R})$. Defining $[\psi]^{\varepsilon}$ by $[\psi]^{\varepsilon}(x)=\psi\left(\frac{x}{\varepsilon}\right)$, then

$$
[\psi]^{\varepsilon} \rightarrow \int_{0}^{1} \psi(\xi) d \xi \text { in } L^{\infty}(\mathbb{R}) \text { weak-* }
$$


Remark 1.3. Convergence (1.24) means that for any function $\phi \in L^{1}(\mathbb{R})$,

$$
\int_{\mathbb{R}}[\psi]^{\varepsilon}(x) \phi(x) d x \rightarrow \int_{0}^{1} \psi(\xi) d \xi \int_{\mathbb{R}} \phi(x) d x .
$$

Proof. The first step of the proof consists in noticing that, since the space $\mathcal{D}(\mathbb{R})$ is dense in $L^{1}(\mathbb{R})$, it is enough to prove

$$
\int_{\mathbb{R}}[\psi]^{\varepsilon}(x) \varphi(x) d x \rightarrow \int_{0}^{1} \psi(\xi) d \xi \int_{\mathbb{R}} \varphi(x) d x
$$

for any fixed $\varphi \in \mathcal{D}(\mathbb{R})$.

In a second step, fixing a $\mathcal{D}(\mathbb{R})$-function $\varphi$, a real $M$ such that $[-M, M]$ contains the support of $\varphi$ is chosen. Then, the set of points $\{-M,-M+\varepsilon,-M+2 \varepsilon, \ldots, M+\mathbb{E}(2 M / \varepsilon) \varepsilon, M+(\mathbb{E}(2 M / \varepsilon)+1) \varepsilon\}$, where $\mathbb{E}$ stands for the integer part, is considered and the integral in 1.26 is shared in the following way:

$$
\int_{\mathbb{R}}[\psi]^{\varepsilon}(x) \varphi(x) d x=\sum_{i=1}^{\mathbb{E}(2 M / \varepsilon)+1} \int_{-M+(i-1) \varepsilon}^{-M+i \varepsilon} \psi\left(\frac{x}{\varepsilon}\right) \varphi(x) d x .
$$

In the third step, since $\varphi$ is regular, it is possible to write that, for any $i=1, \ldots, \mathbb{E}(2 M / \varepsilon)+1$, for any $x \in[-M+(i-1) \varepsilon,-M+i \varepsilon]$, there exists a $c_{i}(x) \in[-M+(i-1) \varepsilon, x]$ such that $\varphi(x)=$ $\varphi(-M+(i-1) \varepsilon)+(x+M-(i-1) \varepsilon) \varphi^{\prime}\left(c_{i}(x)\right)$. Clearly,

$$
|(x+M-(i-1) \varepsilon)| \leq \varepsilon \text { and }\left|\varphi^{\prime}(c(x))\right| \leq\left\|\varphi^{\prime}\right\|_{\infty}
$$

Using this in the sum of 1.27 yields

$$
\begin{aligned}
\int_{\mathbb{R}}[\psi]^{\varepsilon}(x) \varphi(x) d x= & \sum_{i=1}^{\mathbb{E}(2 M / \varepsilon)+1} \int_{-M+(i-1) \varepsilon}^{-M+i \varepsilon} \psi\left(\frac{x}{\varepsilon}\right) d x \varphi(-M(i-1) \varepsilon) \\
& +\sum_{i=1}^{\mathbb{E}(2 M / \varepsilon)+1} \int_{-M+(i-1) \varepsilon}^{-M+i \varepsilon} \psi\left(\frac{x}{\varepsilon}\right)(x+M-(i-1) \varepsilon) \varphi^{\prime}\left(c_{i}(x)\right) d x
\end{aligned}
$$

In the last step, using the periodicity of $\psi$, the first term of 1.29 becomes

$$
\int_{0}^{1} \psi(\xi) d \xi \varepsilon \sum_{i=1}^{\mathbb{E}(2 M / \varepsilon)+1} \varphi(-M(i-1) \varepsilon),
$$

which, because of Riemann like definitions of an integral, converges towards

$$
\int_{0}^{1} \psi(\xi) d \xi \int_{\mathbb{R}} \varphi(x) d x
$$

as $\varepsilon$ goes to 0 . Beside this using 1.28 , the second term of 1.29 is bounded in the following way:

$$
\begin{aligned}
\left|\sum_{i=1}^{\mathbb{E}(2 M / \varepsilon)+1} \int_{-M+(i-1) \varepsilon}^{-M+i \varepsilon} \psi\left(\frac{x}{\varepsilon}\right)(x+M-(i-1) \varepsilon) \varphi^{\prime}\left(c_{i}(x)\right) d x\right| \\
\leq \int_{0}^{1}|\psi(\xi)| \varepsilon d \xi\left(\frac{2 M+1}{\varepsilon}\right) \varepsilon\left\|\varphi^{\prime}\right\|_{\infty} \leq \varepsilon(2 M+1) \int_{0}^{1}|\psi(\xi)| d \xi\left\|\varphi^{\prime}\right\|_{\infty}
\end{aligned}
$$


and then converges to 0 as $\varepsilon$ goes to 0 .

A careful watch to 1.27, 1.31 and 1.32 gives convergence 1.25 . Since, this can be done for any $\varphi \in \mathcal{D}(\mathbb{R})$, the Lemma is proved.

In view of Lemma 1.2 and from the application point of view, it is cleaver to see the $L^{\infty}(\mathbb{R})$ weak-* convergence as a way to generalize the concept of average value to functions which have non-periodic oscillations.

Hence finding $\mathcal{H}$ involved in 1.4 - or other similar question - may be translated into a mathematical framework as: "Find an equation satisfied by the weak-* limit of $u^{\varepsilon} . "$

This explains why weak-* limit is a key-notion of Homogenization Theory

The second result characterizes the asymptotic behavior of a function depending on $x$ and $\xi$, with a periodic dependence in $\xi$, and applied in $\xi=x / \varepsilon$. Notice that here more regularity with respect to $\xi$ is needed than previously.

Lemma 1.4. Let $\psi=\psi(x, \xi) \in L^{\infty}\left(\mathbb{R} ; \mathcal{C}_{\#}^{0}(\mathbb{R})\right)$ and define $[\psi]^{\varepsilon}$ by setting $[\psi]^{\varepsilon}(x)=\psi\left(x, \frac{x}{\varepsilon}\right)$. Then

$$
[\psi]^{\varepsilon} \rightarrow \int_{0}^{1} \psi(x, \xi) d \xi \text { in } L^{\infty}(\mathbb{R}) w_{e a k-*}
$$

Remark 1.5. It is not completely obvious that the limit function in $(1.33)$ is in $L^{\infty}\left(\mathbb{R} ; \mathcal{C}_{\#}^{0}(\mathbb{R})\right)$. This is insured by the fact that $\psi$ is continuous with respect to $\xi$. More details on that are given in subsection 2.3.

On another hand, convergence (1.33) means that for any function $\phi \in L^{1}(\mathbb{R})$,

$$
\int_{\mathbb{R}}[\psi]^{\varepsilon}(x) \phi(x) d x \rightarrow \int_{\mathbb{R}}\left(\int_{0}^{1} \psi(x, \xi) d \xi\right) \phi(x) d x .
$$

But, for the same reason as previously, if the following convergence

$$
\int_{\mathbb{R}}[\psi]^{\varepsilon}(x) \varphi(x) d x \rightarrow \int_{\mathbb{R}}\left(\int_{0}^{1} \psi(x, \xi) d \xi\right) \varphi(x) d x
$$

is proved for any $\varphi \in \mathcal{D}(\mathbb{R})$, it gives the Lemma.

Proof. (I chose to restrict the proof to the case when $\psi \in \mathcal{C}^{0}\left(\mathbb{R} ; \mathcal{C}_{\#}^{0}(\mathbb{R})\right)$ to avoid technical arguments linked with Integration Theory.) The first stage of this proof consists in partitioning interval $[0,1]$ into $m$ intervals of length $1 / m$, for any integer $m$. Then, fixing any value of $m$, the characteristic functions $\chi_{i}$, for $i=1 \ldots, m$, of all intervals are considered. They are extended by periodicity over $\mathbb{R}$. The center $\xi_{i}$ of each interval is also considered. Using them, function $\tilde{\psi}_{m}$ defined by

$$
\tilde{\psi}_{m}(x, \xi)=\sum_{i=1}^{m} \psi\left(x, \xi_{i}\right) \chi_{i}(\xi)
$$

is built. For every $x, \xi \mapsto \tilde{\psi}_{m}(x, \xi)$ is constant by intervals and, as $m$ tends to infinity,

$$
\tilde{\psi}_{m}(x, \xi) \rightarrow \psi(x, \xi) \quad \text { uniformly on every compact of } \mathbb{R}^{2} \text {. }
$$

Since, applying Lemma 1.2 .

$$
\left[\chi_{i}\right]^{\varepsilon} \rightarrow \int_{0}^{1} \chi(\xi) d \xi=\frac{1}{m} \text { in } L^{\infty}(\mathbb{R}) \text { weak-* }
$$


it may be gotten:

$$
\left[\tilde{\psi}_{m}\right]^{\varepsilon} \rightarrow \sum_{i=1}^{m} \psi\left(x, \xi_{i}\right) \frac{1}{m} \text { in } L^{\infty}(\mathbb{R}) \text { weak-* }
$$

which is clearly $\int_{0}^{1} \tilde{\psi}_{m}(x, \xi) d \xi$. Hence, as $\varepsilon$ goes to 0 ,

$$
\left[\tilde{\psi}_{m}\right]^{\varepsilon} \rightarrow \int_{0}^{1} \tilde{\psi}_{m}(x, \xi) d \xi \text { in } L^{\infty}(\mathbb{R}) \text { weak-* }
$$

The second stage of the proof consists in fixing $\varphi \in \mathcal{D}(\mathbb{R})$, and in showing that for any $\delta>0$, it is possible find an $\varepsilon_{0}$, such that for any $\varepsilon \leq \varepsilon_{0}$,

$$
\left|\int_{\mathbb{R}}[\psi]^{\varepsilon}(x) \varphi(x) d x-\int_{\mathbb{R}}\left(\int_{0}^{1} \psi(x, \xi) d \xi\right) \varphi(x) d x\right| \leq \delta .
$$

The way to get this inequality consists in writing :

$$
\begin{aligned}
& \left|\int_{\mathbb{R}}[\psi]^{\varepsilon}(x) \varphi(x) d x-\int_{\mathbb{R}}\left(\int_{0}^{1} \psi(x, \xi) d \xi\right) \varphi(x) d x\right|= \\
& \mid \int_{\mathbb{R}}\left([\psi]^{\varepsilon}(x)-\left[\tilde{\psi}_{m}\right]^{\varepsilon}(x)\right) \varphi(x) d x+\int_{\mathbb{R}}\left(\left[\tilde{\psi}_{m}\right]^{\varepsilon}(x)-\int_{0}^{1} \tilde{\psi}_{m}(x, \xi) d \xi\right) \varphi(x) d x \\
& +\int_{\mathbb{R}}\left(\int_{0}^{1} \tilde{\psi}_{m}(x, \xi) d \xi-\int_{0}^{1} \psi(x, \xi) d \xi\right) \varphi(x) d x \mid \leq \\
& \int_{\mathbb{R}}\left|[\psi]^{\varepsilon}(x)-\left[\tilde{\psi}_{m}\right]^{\varepsilon}(x)\right| \varphi(x)|d x+| \int_{\mathbb{R}}\left(\left[\tilde{\psi}_{m}\right]^{\varepsilon}(x)-\int_{0}^{1} \tilde{\psi}_{m}(x, \xi) d \xi\right) \varphi(x) d x \mid \\
& \quad+\int_{\mathbb{R}}\left(\int_{0}^{1}\left|\tilde{\psi}_{m}(x, \xi)-\psi(x, \xi)\right| d \xi\right)|\varphi(x)| d x
\end{aligned}
$$

Because of uniform convergence 1.37), it is possible to fix an $m$ such that

$$
\begin{gathered}
\int_{\mathbb{R}}\left|[\psi]^{\varepsilon}(x)-\left[\tilde{\psi}_{m}\right]^{\varepsilon}(x)\right||\varphi(x)| d x \leq \frac{\delta}{3} \text { for any } \varepsilon, \\
\int_{\mathbb{R}}\left(\int_{0}^{1}\left|\tilde{\psi}_{m}(x, \xi)-\psi(x, \xi)\right| d \xi\right)|\varphi(x)| d x \leq \frac{\delta}{3},
\end{gathered}
$$

and once this $m$ is fixed, because of 1.40 , it is possible to fix an $\varepsilon_{0}$ such that

$$
\left|\int_{\mathbb{R}}\left(\left[\tilde{\psi}_{m}\right]^{\varepsilon}(x)-\int_{0}^{1} \tilde{\psi}_{m}(x, \xi) d \xi\right) \varphi(x) d x\right| \leq \frac{\delta}{3} \text { for any } \varepsilon \leq \varepsilon_{0} .
$$

Using 1.43, 1.43 and 1.45 in 1.42 gives the sought formula 1.41 .

Since, this can be done for any $\varphi \in \mathcal{D}(\mathbb{R})$, the Lemma is proved. 


\section{Two-Scale Convergence - Definition and Results}

\subsection{Definitions}

There are several variants of the Two-Scale Convergence result, more or less well adapted to targeted applications and involving various functional spaces (see Nguetseng [27, 28, Allaire [3, Amar 4], Casado-Díaz \& Gayte 8, Frénod, Raviart \& Sonnendrücker 18], Nguetseng \& Woukeng [30] and Nguetseng \& Svanstedt [29]). They are in fact very close to each other in what concerns what they claim and their proofs all follow the same routine based on these two phases :

- A continuous injection Lemma,

- A compactness Theorem,

Remark 2.1. In 2005, Pak [31] made a important improvement in the Two-Scale Convergence Theory adapting it to manifolds and differential forms.

I have chosen to present the Two-Scale Convergence in the framework set out in Frénod, Raviart \& Sonnendrücker [18] since this framework permits to select among the variables the ones that carry oscillations and the others.

I begin by giving some notations.

Definition 2.2. Let $\Omega$ be a regular domain in $\mathbb{R}^{n}$, let $\mathcal{L}$ be one of the following functional spaces: Lebesgue space $L^{r}(O)$ for $r \in[1,+\infty)$ and $O$ a regular manifold or Sobolev space $W^{l, m}(O)$ for $l \in \mathbb{N}$ and $m \in[1,+\infty)$; and, $\mathcal{L}^{\prime}$ its topological dual space. Let $q \in[1,+\infty)$ and $p \in(1,+\infty]$ being such that $1 / q+1 / p=1$. Let $\mathcal{C}_{\#}^{0}\left(\mathbb{R}^{n} ; \mathcal{L}\right)$ be the space of continuous functions $\mathbb{R}^{n} \rightarrow \mathcal{L}$ and periodic of period 1 with respect to every variable. Let $L^{p}\left(\Omega, \mathcal{L}^{\prime}\right)$ be the Lebesgue space of Bochner integrable $\mathcal{L}^{\prime}$-valued functions. It can be characterized as the space of (equivalence classes for the equivalence relation "= a.e." of measurable) functions $f: \Omega \rightarrow \mathcal{L}^{\prime}$ such that the $p$-th power of the norm of $f:|f|_{\mathcal{L}^{\prime}}^{p}$ is Lebesgue integrable if $p<\infty$ and such that its norm is essentially bounded if $p=\infty$. Let $L_{\#}^{p}\left(\mathbb{R}^{n} ; \mathcal{L}^{\prime}\right)$ be the Lebesgue space of locally Bochner integrable $\mathcal{L}^{\prime}$-valued functions (it can be characterized as the space of functions $f: \mathbb{R}^{n} \rightarrow \mathcal{L}^{\prime}$ such that the $p$-th power of the norm of $f:|f|_{\mathcal{L}^{\prime}}^{p}$ is locally Lebesgue integrable if $p<\infty$ and such that its norm is locally essentially bounded if $p=\infty$ ) and periodic of period 1. $\left(L_{\#}^{p}\left(\mathbb{R}^{n} ; \mathcal{L}^{\prime}\right)=\left(L_{\#}^{q}\left(\mathbb{R}^{n} ; \mathcal{L}\right)\right)^{\prime}\right.$ las a consequence of the separability of $\mathcal{L}$.) Spaces $L^{q}\left(\Omega ; L_{\#}^{q}\left(\mathbb{R}^{n}, \mathcal{L}\right)\right), L^{q}\left(\Omega ; \mathcal{C}_{\#}^{0}\left(\mathbb{R}^{n} ; \mathcal{L}\right)\right)$ and $L^{p}\left(\Omega ; L_{\#}^{p}\left(\mathbb{R}^{n}, \mathcal{L}^{\prime}\right)\right)$ are also considered. (I refer the reader to Bochner [6] Diestel Uhl [10] Yosida [39] Schwartz [34] for the Theory that allows a precise definition of those spaces.)

Remark 2.3. Space $\mathcal{L}$ can be a more general separable Banach space than evoked in Definition 2.2. Nonetheless, it cannot be any Banach space. It has to be chosen such that its dual space $\mathcal{L}^{\prime}$ has the Radon-Nikodym Property (see for instance [10, Theorem 1, Chapter 4]).

Now, I give the definition of Two-Scale Convergence.

Definition 2.4. A sequence $\left(u^{\varepsilon}\right)=\left(u^{\varepsilon}(\mathbf{x})\right) \subset L^{p}\left(\Omega ; \mathcal{L}^{\prime}\right)$ is said to Two-Scale converge to a profile $U=U(\mathbf{x}, \boldsymbol{\xi}) \in L^{p}\left(\Omega ; L_{\#}^{p}\left(\mathbb{R}^{n}, \mathcal{L}^{\prime}\right)\right)$ if, for any function $\phi=\phi(\mathbf{x}, \boldsymbol{\xi}) \in L^{q}\left(\Omega ; \mathcal{C}_{\#}^{0}\left(\mathbb{R}^{n} ; \mathcal{L}\right)\right)$, the following convergence holds:

$$
\lim _{\varepsilon \rightarrow 0} \int_{\Omega} \mathcal{L}^{\prime}\left\langle u^{\varepsilon}(\mathbf{x}), \phi\left(\mathbf{x}, \frac{\mathbf{x}}{\varepsilon}\right)\right\rangle_{\mathcal{L}} d \mathbf{x}=\int_{\Omega} \int_{[0,1]^{n}} \mathcal{L}^{\prime}\langle U(\mathbf{x}, \boldsymbol{\xi}), \phi(\mathbf{x}, \boldsymbol{\xi})\rangle_{\mathcal{L}} d \mathbf{x} d \boldsymbol{\xi}
$$

where $\mathcal{L}^{\prime}\langle.,\rangle_{\mathcal{L}}$ is the duality bracket between $\mathcal{L}^{\prime}$ and $\mathcal{L}$.

Remark 2.5. Despite it is not obvious, the fact that $\phi\left(\mathbf{x}, \frac{\mathbf{x}}{\varepsilon}\right)$ is well in $L^{q}(\Omega ; \mathcal{L})$ is true. This question is tackled in the sequel (see Lemma 2.10).

To end this definition subsection, I give two definitions of the Strong Two-Scale Convergence. 
Definition 2.6. If $p=q=2, \mathcal{L}$ is a Hilbert space and $U \in L^{2}\left(\Omega ; \mathcal{C}_{\#}^{0}\left(\mathbb{R}^{n} ; \mathcal{L}^{\prime}\right)\right)$, the sequence $\left(u^{\varepsilon}\right)=\left(u^{\varepsilon}(\mathbf{x})\right) \subset L^{2}\left(\Omega ; \mathcal{L}^{\prime}\right)$ is said to Strongly Two-Scale convergence to $U=U(\mathbf{x}, \boldsymbol{\xi})$ if

$$
\lim _{\varepsilon \rightarrow 0} \int_{\Omega}\left|u^{\varepsilon}(\mathbf{x})-U\left(\mathbf{x}, \frac{\mathbf{x}}{\varepsilon}\right)\right|_{\mathcal{L}^{\prime}}^{2} d \mathbf{x}=0 .
$$

In (2.2) $|.|_{\mathcal{L}^{\prime}}^{2}$ is the norm in $\mathcal{L}^{\prime}-$ which can be identified with $\mathcal{L}$ - associated with inner product $\mathcal{L}^{\prime}\langle., .\rangle_{\mathcal{L}^{\prime}}$ of $\mathcal{L}^{\prime}-$ which is also the inner product ${ }_{\mathcal{L}}\langle.,\rangle_{\mathcal{L}}$ of $\mathcal{L}$ and the duality bracket $\mathcal{L}^{\prime}\langle.,\rangle_{\mathcal{L}}$ between $\mathcal{L}^{\prime}$ and $\mathcal{L}$.

In Definition 2.6, the assumption that $U$ is continuous with respect to $\boldsymbol{\xi}$ is to insure the ability to compute $U(\mathbf{x}, \mathbf{x} / \varepsilon)$, which is not insured for a function which is defined only almost everywhere, since the measure of $\left\{(\mathbf{x}, \mathbf{x} / \varepsilon), \mathbf{x} \in \mathbb{R}^{n}\right\}$ is 0 in $\mathbb{R}^{n} \times \mathbb{R}^{n}$. Nguetseng \& Woukeng [30] gave a definition of Strong Two-Scale Convergence which involves less regularity:

Definition 2.7. If $p=q=2, \mathcal{L}$ is a Hilbert space and $U \in L^{2}\left(\Omega ; L_{\#}^{2}\left(\mathbb{R}^{n} ; \mathcal{L}^{\prime}\right)\right)$, the sequence $\left(u^{\varepsilon}\right)=\left(u^{\varepsilon}(\mathbf{x})\right) \subset L^{2}\left(\Omega ; \mathcal{L}^{\prime}\right)$ is said to Strongly Two-Scale convergence to $U=U(\mathbf{x}, \boldsymbol{\xi})$ if

$$
\begin{gathered}
\forall \delta>0, \exists \varepsilon_{0} \text { and } \tilde{U} \in L^{2}\left(\Omega ; \mathcal{C}_{\#}^{0}\left(\mathbb{R}^{n} ; \mathcal{L}^{\prime}\right)\right) \text { such that } \\
\int_{\Omega} \int_{[0,1]^{n}}|U(\mathbf{x}, \boldsymbol{\xi})-\tilde{U}(\mathbf{x}, \boldsymbol{\xi})|_{\mathcal{L}^{\prime}}^{2} d \mathbf{x} d \boldsymbol{\xi} \leq \frac{\delta}{2}, \text { and } \\
\int_{\Omega}\left|u^{\varepsilon}(\mathbf{x})-\tilde{U}\left(\mathbf{x}, \frac{\mathbf{x}}{\varepsilon}\right)\right|_{\mathcal{L}^{\prime}}^{2} d \mathbf{x} \leq \frac{\delta}{2}, \text { for every } \varepsilon \leq \varepsilon_{0} .
\end{gathered}
$$

\subsection{Link with Weak Convergence}

Two-Scale Convergence and weak-* convergence are strongly related. In fact Two-Scale Convergence may be seen as a generalization of weak-* convergence. This link expresses by the following Proposition.

Proposition 2.8. If a sequence $\left(u^{\varepsilon}\right) \subset L^{p}\left(\Omega ; \mathcal{L}^{\prime}\right)$ Two-Scale converges to $U \in L^{p}\left(\Omega ; L_{\#}^{p}\left(\mathbb{R}^{n} ; \mathcal{L}^{\prime}\right)\right)$, then

$$
u^{\varepsilon}-\int_{[0,1]^{n}} U(., \boldsymbol{\xi}) d \boldsymbol{\xi} \text { weak-* in } L^{p}\left(\Omega ; \mathcal{L}^{\prime}\right)
$$

Proof. Choosing test functions $\phi(\mathbf{x}, \boldsymbol{\xi})=\phi(\mathbf{x})$ - independent of the oscillating variable $\boldsymbol{\xi}$ - for any $\phi \in$ $\left.L^{q}(\Omega ; \mathcal{L})\right)$ in the definition of Two-Scale Convergence is possible since $\left.L^{q}(\Omega ; \mathcal{L})\right) \subset L^{q}\left(\Omega ; \mathcal{C}_{\#}^{0}\left(\mathbb{R}^{n} ; \mathcal{L}\right)\right)$. Doing this yields:

$$
\begin{aligned}
\lim _{\varepsilon \rightarrow 0} \int_{\Omega} \mathcal{L}^{\prime}\left\langle u^{\varepsilon}(\mathbf{x}), \phi(\mathbf{x})\right\rangle_{\mathcal{L}} d \mathbf{x}=\int_{\Omega} \int_{[0,1]^{n}} \mathcal{L}^{\prime}\langle U(\mathbf{x}, \boldsymbol{\xi}), \phi(\mathbf{x})\rangle_{\mathcal{L}} d \mathbf{x} d \boldsymbol{\xi}= \\
\qquad \int_{\Omega} \mathcal{L}^{\prime}\left\langle\left(\int_{[0,1]^{n}} U(\mathbf{x}, \boldsymbol{\xi}) d \boldsymbol{\xi}\right), \phi(\mathbf{x})\right\rangle_{\mathcal{L}} d \mathbf{x}
\end{aligned}
$$

which is nothing but 2.4, proving the Proposition.

Remark 2.9. The last equality in 2.5) can be considered as trivial and is a consequence of Hille Theorem. Nevertheless, I give in this Remark the details bringing it.

For any fixed integer $m$, a partition of a subdomain $\omega_{m}$ of $\Omega$ with $m K(m)$ hypercubes of measure $1 / m$ and a partition of $[0,1]^{n}$ with $m$ hypercubes of measure $1 / m$ are considered. If $\Omega$ is compact, $\omega_{m}=\Omega$ for every $m$ and $K(m)$ is constant and equal to the measure of $\Omega$; if $\Omega$ is not compact, $\left(\omega_{m}\right)$ is a sequence of subdomains such that $\omega_{m} \subset \omega_{m+1}$ for every $m$, such that the measure of $\omega_{m}$ 
is $K(m)<+\infty$ and $\cup_{m \in \mathbb{N}} \omega_{m}=\Omega$.

$\phi_{k}$, for $k=1, \ldots, m K(m)$, stands for the value on the $k$-th hypercube of a piecewise constant function approximating $\phi$ and $U_{k, l}$, for $k=1, \ldots, m K(m)$ and $l=1, \ldots, m$, is the value on the tensor product of the $k$-th hypercube of $\omega_{m}$ and the $l$-th hypercube of $[0,1]^{n}$ of a piecewise constant function approximating $U$.

$$
\begin{aligned}
& \int_{\Omega} \int_{[0,1]^{n}} \mathcal{L}^{\prime}\langle U(\mathbf{x}, \boldsymbol{\xi}), \phi(\mathbf{x})\rangle_{\mathcal{L}} d \mathbf{x} d \boldsymbol{\xi}=\lim _{m \rightarrow+\infty} \sum_{k=1}^{m K(m)} \sum_{=1}^{m} \frac{1}{m^{2}} \mathcal{L}^{\prime}\left\langle U_{k, l}, \phi_{k}\right\rangle_{\mathcal{L}}= \\
& \lim _{m \rightarrow+\infty} \sum_{k=1}^{m K(m)} \frac{1}{m} \mathcal{L}^{\prime}\left\langle\left(\frac{1}{m} \sum_{=1}^{m} U_{k, l}\right), \phi_{k}\right\rangle_{\mathcal{L}}=\int_{\Omega} \mathcal{L}^{\prime}\left\langle\left(\int_{[0,1]^{n}} U(\mathbf{x}, \boldsymbol{\xi}) d \boldsymbol{\xi}\right), \phi(\mathbf{x})\right\rangle_{\mathcal{L}} d \mathbf{x} .
\end{aligned}
$$

\subsection{Injection Lemma}

Now, I turn to the first important ingredient of Two-Scale Convergence which is the fact that taking functions of $L^{q}\left(\Omega ; \mathcal{C}_{\#}^{0}\left(\mathbb{R}^{n}, \mathcal{L}\right)\right)$ in $\boldsymbol{\xi}=\mathbf{x} / \varepsilon$ is a way to inject continuously this space in $L^{q}(\Omega ; \mathcal{L})$.

Lemma 2.10. If $\phi \in L^{q}\left(\Omega ; \mathcal{C}_{\#}^{0}\left(\mathbb{R}^{n} ; \mathcal{L}\right)\right)$, then for all $\varepsilon>0$, function $[\phi]^{\varepsilon}: \Omega \rightarrow \mathcal{L}$ defined by

$$
[\phi]^{\varepsilon}(\mathbf{x})=\phi\left(\mathbf{x}, \frac{\mathbf{x}}{\varepsilon}\right)
$$

is measurable and satisfies

$$
\left\|[\phi]^{\varepsilon}\right\|_{L^{q}(\Omega ; \mathcal{L})} \leq\|\phi\|_{L^{q}\left(\Omega ; \mathcal{C}_{\#}^{0}\left(\mathbb{R}^{n} ; \mathcal{L}\right)\right)}
$$

Proof. The first point is to see that $\phi \in L^{q}\left(\Omega ; \mathcal{C}_{\#}^{0}\left(\mathbb{R}^{n} ; \mathcal{L}\right)\right)$ if and only if there exists a set $E$ of measure zero in $\Omega$ such that

$$
\begin{aligned}
& \forall \mathbf{x} \in \Omega \backslash E, \boldsymbol{\xi} \mapsto \phi(\mathbf{x}, \boldsymbol{\xi}) \text { is continuous and periodic, } \\
& \forall \boldsymbol{\xi} \in[0,1]^{n}, \mathbf{x} \mapsto \phi(\mathbf{x}, \boldsymbol{\xi}) \text { is measurable over } \Omega, \\
& \mathbf{x} \mapsto \sup _{\boldsymbol{\xi} \in[0,1]^{n}}|\phi(\mathbf{x}, \boldsymbol{\xi})|_{\mathcal{L}} \text { is } L^{q}\left(\Omega ; \mathbb{R}^{+}\right) .
\end{aligned}
$$

Remark 2.11. This equivalence property is a consequence of Bochner integration theory but is not completely obvious. Nevertheless, I take it for granted. In this Remark, I just recall milestones that are needed to get it. For details, I refer to Diestel \& Uhl [10], Yosida [39] and Schwartz [34].

Denoting $L^{q}\left(\Omega ; \mathcal{C}_{\#}^{0}\left(\mathbb{R}^{n} ; \mathcal{L}\right)\right)$ means that measured space $[\Omega$, completed of Borelian $\sigma-$ algebra, Lebesgue measure] in considered.

Since $\mathcal{L}$ is a separable Banach space, $\mathcal{C}_{\#}^{0}\left(\mathbb{R}^{n} ; \mathcal{L}\right)$ is also a separable Banach space. Hence, the following measurable space $\left[\mathcal{C}_{\#}^{0}\left(\mathbb{R}^{n} ; \mathcal{L}\right)\right.$, Borelian $\sigma$-algebra $]$ may be considered.

Then, the use of the definition of strongly measurable function in the sense of Bochner $\Omega \rightarrow$ $\mathcal{C}_{\#}^{0}\left(\mathbb{R}^{n} ; \mathcal{L}\right)$ is needed. (A function $f$ is strongly measurable in the sense of Bochner if there exists a set $E^{\prime}$ of measure zero in $\Omega$ such that $f\left(\Omega \backslash E^{\prime}\right)$ is included in a separable subset of $\mathcal{C}_{\#}^{0}\left(\mathbb{R}^{n} ; \mathcal{L}\right)$, and, if the inverse image by $f$ of any set of the $\sigma$-algebra of $\mathcal{C}_{\#}^{0}\left(\mathbb{R}^{n}, \mathcal{L}\right)$ belongs to $\sigma$-algebra of $\Omega$.)

With this, the integration theory can be implemented, involving step functions $\Omega \rightarrow \mathcal{C}_{\#}^{0}\left(\mathbb{R}^{n}, \mathcal{L}\right)$ (which is quite long) and characterizations of integrable functions are gotten. Among them, there is the following one: A function $f: \Omega \rightarrow \mathcal{C}_{\#}^{0}\left(\mathbb{R}^{n} ; \mathcal{L}\right)$ is integrable if it is strongly measurable in the sense of Bochner and if

$$
\int_{\Omega}\|f\|_{\mathcal{C}_{\#}^{0}\left(\mathbb{R}^{n} ; \mathcal{L}\right)} d \mathbf{x}<+\infty .
$$


Finally, spaces $\mathcal{L}^{p}\left(\Omega ; \mathcal{C}_{\#}^{0}\left(\mathbb{R}^{n} ; \mathcal{L}\right)\right)$ and $L^{q}\left(\Omega ; \mathcal{C}_{\#}^{0}\left(\mathbb{R}^{n} ; \mathcal{L}\right)\right)$ can be built. Characterization of $L^{q}(\Omega ;$ $\mathcal{C}_{\#}^{0}\left(\mathbb{R}^{n} ; \mathcal{L}\right)$ by 2.9), 2.10 and 2.11) can also be gotten.

The second point consists in fixing $\varepsilon$. From 2.9 , for all fixed $\varepsilon,[\phi]^{\varepsilon}(\mathbf{x})=\phi\left(\mathbf{x}, \frac{\mathbf{x}}{\varepsilon}\right)$ is well defined on $\Omega \backslash E$.

Now, the goal is to prove that $[\phi]^{\varepsilon}$ is measurable. For this purpose, for any fixed integer $m$, a partition of a subdomain $\omega_{m}$ of $\Omega$ with $m K(m)$ hypercubes of measure $1 / m$ is considered. (If $\Omega$ is compact, $\omega_{m}=\Omega$ for every $m$ and $K(m)$ is constant and equal to the measure of $\Omega$; if $\Omega$ is not compact, $\left(\omega_{m}\right)$ is a sequence of subdomains such that $\omega_{m} \subset \omega_{m+1}$ for every $m$, such that the measure of $\omega_{m}$ is $K(m)$ and $\cup_{m \in \mathbb{N}} \omega_{m}=\Omega$.)

For every $i=1, \ldots, m K(m)$, the center $\mathbf{x}_{i}$ of the $i$-th hypercube and the characteristic function $\iota_{i}$ of the $i$-th hypercube are considered. With this, function $\boldsymbol{\eta}_{m}^{\varepsilon}$ defined by

$$
\boldsymbol{\eta}_{m}^{\varepsilon}(\mathbf{x})=\sum_{i=1}^{m K(m)} \frac{\mathbf{x}_{i}}{\varepsilon} \iota_{i}(\mathbf{x})
$$

is a step function approximating $\frac{\mathbf{x}}{\varepsilon}$.

Considering now function $[\phi]_{m}^{\varepsilon}$ defined by

$$
[\phi]_{m}^{\varepsilon}(\mathbf{x})=\phi\left(\mathbf{x}, \boldsymbol{\eta}_{m}^{\varepsilon}(\mathbf{x})\right)
$$

clearly,

$$
\forall \mathbf{x} \in \Omega \backslash E,[\phi]_{m}^{\varepsilon}(\mathbf{x}) \rightarrow[\phi]^{\varepsilon}(\mathbf{x}) \text { as } m \rightarrow+\infty
$$

and, since

$$
[\phi]_{m}^{\varepsilon}(\mathbf{x})=\phi\left(\mathbf{x}, \boldsymbol{\eta}_{m}^{\varepsilon}(\mathbf{x})\right)=\sum_{i=1}^{m K(m)} \phi\left(\mathbf{x}, \frac{\mathbf{x}_{i}}{\varepsilon}\right) \iota_{i}(\mathbf{x}),
$$

and because of $[2.10),[\phi]_{m}^{\varepsilon}$ is a finite sum of measurable functions, hence is measurable. Hence, $[\phi]^{\varepsilon}$ is almost everywhere the limit of a measurable function. Hence it is measurable.

The last point is the proof of 2.8). From 2.11) it is deduced that

$$
\|\phi\|_{L^{q}\left(\Omega ; \mathcal{C}_{\#}^{0}\left(\mathbb{R}^{n} ; \mathcal{L}\right)\right)}^{q}=\int_{\Omega}\left(\sup _{\boldsymbol{\xi} \in[0,1]^{n}}|\phi(\mathbf{x}, \boldsymbol{\xi})|_{\mathcal{L}}\right)^{q} d \mathbf{x}<+\infty .
$$

On the other hand

$$
\left\|[\phi]^{\varepsilon}\right\|_{L^{q}(\Omega ; \mathcal{L})}=\int_{\Omega}\left|\phi\left(\mathbf{x}, \frac{\mathbf{x}}{\varepsilon}\right)\right|_{\mathcal{L}}^{q} d \mathbf{x} \leq \int_{\Omega}\left(\sup _{\boldsymbol{\xi} \in[0,1]^{n}}|\phi(\mathbf{x}, \boldsymbol{\xi})|_{\mathcal{L}}\right)^{q} d \mathbf{x}=\|\phi\|_{L^{q}\left(\Omega ; \mathcal{C}_{\#}^{0}\left(\mathbb{R}^{n} ; \mathcal{L}\right)\right)}^{q},
$$

which is 2.8, ending the proof of the Lemma.

This Injection Lemma is supplemented by a property giving information on the asymptotic behavior of $[\phi]^{\varepsilon}$ as $\varepsilon$ goes to zero. 
Proposition 2.12. Under the same assumption as in Lemma 2.10, function $[\phi]^{\varepsilon}$ defined by 2.7) satisfies:

$$
\lim _{\varepsilon \rightarrow 0}\left\|[\phi]^{\varepsilon}\right\|_{L^{q}(\Omega ; \mathcal{L})}^{q}=\lim _{\varepsilon \rightarrow 0} \int_{\Omega}\left|\phi\left(\mathbf{x}, \frac{\mathbf{x}}{\varepsilon}\right)\right|_{\mathcal{L}}^{q} d \mathbf{x}=\int_{\Omega} \int_{[0,1]^{n}}|\phi(\mathbf{x}, \boldsymbol{\xi})|_{\mathcal{L}}^{q} d \mathbf{x} d \boldsymbol{\xi}=\|\phi\|_{L^{q}\left(\Omega ; L_{\#}^{q}\left(\mathbb{R}^{n} ; \mathcal{L}\right)\right)}^{q} .
$$

Proof. For any fixed integer $m$, a partition of $[0,1]^{n}$ with $m$ hypercubes of measure $1 / m$ is considered. the center of the $i$-th hypercube is called $\boldsymbol{\xi}_{i}$ and its characteristic function, extended by periodicity over $\mathbb{R}^{n}$ is called $\chi_{i}$. With $\phi$ is associated the sequence of function $\tilde{\phi}_{m}$ defined by

$$
\tilde{\phi}_{m}(\mathbf{x}, \boldsymbol{\xi})=\sum_{i=1}^{m} \phi\left(\mathbf{x}, \boldsymbol{\xi}_{i}\right) \chi_{i}(\boldsymbol{\xi})
$$

The second step consists in considering $\left[\chi_{i}\right]^{\varepsilon}$ defined by

$$
\left[\chi_{i}\right]^{\varepsilon}(\mathbf{x})=\chi_{i}\left(\frac{\mathbf{x}}{\varepsilon}\right) .
$$

using Lemma 1.2 generalized to the multidimensional setting, the following is deduced:

$$
\begin{gathered}
{\left[\chi_{i}\right]^{\varepsilon} \rightarrow \int_{[0,1]^{n}} \chi_{i}(\boldsymbol{\xi}) d \boldsymbol{\xi}=\frac{1}{m} \quad \text { in } L^{\infty}(\Omega ; \mathbb{R}) \text { weak-* }} \\
\left(\left[\chi_{i}\right]^{\varepsilon}\right)^{q} \rightarrow \int_{[0,1]^{n}} \chi_{i}^{q}(\boldsymbol{\xi}) d \boldsymbol{\xi}=\frac{1}{m} \quad \text { in } L^{\infty}(\Omega ; \mathbb{R}) \text { weak-*. }
\end{gathered}
$$

Hence

$$
\begin{aligned}
\lim _{\varepsilon \rightarrow 0} \int_{\Omega}\left|\tilde{\phi}_{m}\left(\mathbf{x}, \frac{\mathbf{x}}{\varepsilon}\right)\right|_{\mathcal{L}}^{q} d \mathbf{x}=\lim _{\varepsilon \rightarrow 0} \int_{\Omega} \sum_{i=1}^{m}\left|\phi\left(\mathbf{x}, \boldsymbol{\xi}_{i}\right)\right|_{\mathcal{L}}^{q}\left(\left[\chi_{i}\right]^{\varepsilon}\right)^{q} d \mathbf{x}= \\
\qquad \sum_{i=1}^{m} \frac{1}{m} \int_{\Omega}\left|\phi\left(\mathbf{x}, \boldsymbol{\xi}_{i}\right)\right|_{\mathcal{L}}^{q} d \mathbf{x}=\int_{\Omega} \int_{[0,1]^{n}}\left|\tilde{\phi}_{m}(\mathbf{x}, \boldsymbol{\xi})\right|_{\mathcal{L}}^{q} d \mathbf{x} d \boldsymbol{\xi} .
\end{aligned}
$$

The goal of the third step is to get, as $m \rightarrow 0$,

$$
\tilde{\phi}_{m} \rightarrow \phi \quad \text { in } L^{q}\left(\Omega ; L_{\#}^{q}\left(\mathbb{R}^{n} ; \mathcal{L}\right)\right) .
$$

For this purpose, the function $\Gamma_{m}: \Omega \rightarrow \mathbb{R}$ defined by

$$
\Gamma_{m}(\mathbf{x})=\sup _{\boldsymbol{\xi} \in[0,1]^{n}}\left|\tilde{\phi}_{m}(\mathbf{x}, \boldsymbol{\xi})-\phi(\mathbf{x}, \boldsymbol{\xi})\right|_{\mathcal{L}}^{q},
$$

is used. Since $\boldsymbol{\xi} \mapsto \tilde{\phi}_{m}(\mathbf{x}, \boldsymbol{\xi})-\phi(\mathbf{x}, \boldsymbol{\xi})$ is piecewise continuous,

$$
\sup _{\boldsymbol{\xi} \in[0,1]^{n}}\left|\tilde{\phi}_{m}(\mathbf{x}, \boldsymbol{\xi})-\phi(\mathbf{x}, \boldsymbol{\xi})\right|_{\mathcal{L}}^{q}=\sup _{\boldsymbol{\xi} \in[0,1]^{n} \cap \mathbb{Q}^{n}}\left|\tilde{\phi}_{m}(\mathbf{x}, \boldsymbol{\xi})-\phi(\mathbf{x}, \boldsymbol{\xi})\right|_{\mathcal{L}}^{q} .
$$

Hence, $\Gamma_{m}$ is a supremum on an countable set of measurable functions and, as such, measurable.

Moreover,

$$
\begin{gathered}
\Gamma_{m}(\mathbf{x}) \rightarrow 0 \quad \text { a.e. on } \Omega \\
0 \leq \Gamma_{m}(\mathbf{x}) \leq 2 \sup _{\boldsymbol{\xi} \in[0,1]^{n}}|\phi(\mathbf{x}, \boldsymbol{\xi})|_{\mathcal{L}}^{q},
\end{gathered}
$$


and $\sup _{\boldsymbol{\xi} \in[0,1]^{n}}|\phi(\mathbf{x}, \boldsymbol{\xi})|_{\mathcal{L}}^{q}$ is an integrable function. Hence, invoking the Lebesgue Dominated Convergence Theorem, it may be deduced that, as $m \rightarrow 0$ :

$$
\Gamma_{m}(\mathbf{x}) \rightarrow 0 \quad \text { in } L^{1}(\Omega ; \mathbb{R})
$$

and consequently 2.25$)$.

The last step consists in using (2.24) and 2.25) and writing

$$
\begin{aligned}
& \left\|[\phi]^{\varepsilon}\right\|_{L^{q}(\Omega ; \mathcal{L})}^{q}=\int_{\Omega}\left|\phi\left(\mathbf{x}, \frac{\mathbf{x}}{\varepsilon}\right)\right|_{\mathcal{L}}^{q} d \mathbf{x}=\left(\int_{\Omega}\left|\phi\left(\mathbf{x}, \frac{\mathbf{x}}{\varepsilon}\right)\right|_{\mathcal{L}}^{q} d \mathbf{x}-\int_{\Omega}\left|\tilde{\phi}_{m}\left(\mathbf{x}, \frac{\mathbf{x}}{\varepsilon}\right)\right|_{\mathcal{L}}^{q} d \mathbf{x}\right) \\
& +\left(\int_{\Omega}\left|\tilde{\phi}_{m}\left(\mathbf{x}, \frac{\mathbf{x}}{\varepsilon}\right)\right|_{\mathcal{L}}^{q} d \mathbf{x}-\int_{\Omega} \int_{[0,1]^{n}}\left|\tilde{\phi}_{m}(\mathbf{x}, \boldsymbol{\xi})\right|_{\mathcal{L}}^{q} d \mathbf{x} d \boldsymbol{\xi}\right)+\left(\int_{\Omega} \int_{[0,1]^{n}}\left|\tilde{\phi}_{m}(\mathbf{x}, \boldsymbol{\xi})\right|_{\mathcal{L}}^{q} d \mathbf{x} d \boldsymbol{\xi}\right) .
\end{aligned}
$$

The last term of the right hand side of this formula satisfies

$$
\left(\int_{\Omega} \int_{[0,1]^{n}}\left|\tilde{\phi}_{m}(\mathbf{x}, \boldsymbol{\xi})\right|_{\mathcal{L}}^{q} d \mathbf{x} d \boldsymbol{\xi}\right) \rightarrow\left(\int_{\Omega} \int_{[0,1]^{n}}|\phi(\mathbf{x}, \boldsymbol{\xi})|_{\mathcal{L}}^{q} d \mathbf{x} d \boldsymbol{\xi}\right) \quad \text { as } m \rightarrow+\infty
$$

the second term satisfies

$$
\left(\int_{\Omega}\left|\tilde{\phi}_{m}\left(\mathbf{x}, \frac{\mathbf{x}}{\varepsilon}\right)\right|_{\mathcal{L}}^{q} d \mathbf{x}-\int_{\Omega}\left|\tilde{\phi}_{m}(\mathbf{x}, \boldsymbol{\xi})\right|_{\mathcal{L}}^{q} d \mathbf{x} d \boldsymbol{\xi}\right) \rightarrow 0 \quad \text { as } \varepsilon \rightarrow 0
$$

and concerning the first term,

$$
\begin{aligned}
\left.\left|\int_{\Omega}\right| \phi\left(\mathbf{x}, \frac{\mathbf{x}}{\varepsilon}\right)\right|_{\mathcal{L}} ^{q} d \mathbf{x}-\int_{\Omega}\left|\tilde{\phi}_{m}\left(\mathbf{x}, \frac{\mathbf{x}}{\varepsilon}\right)\right|_{\mathcal{L}}^{q} d \mathbf{x} \mid & \leq\left.\int_{\Omega}|| \phi\left(\mathbf{x}, \frac{\mathbf{x}}{\varepsilon}\right)\right|_{\mathcal{L}} ^{q}-\left|\tilde{\phi}_{m}\left(\mathbf{x}, \frac{\mathbf{x}}{\varepsilon}\right)\right|_{\mathcal{L}}^{q} \mid d \mathbf{x} \\
& \leq \int_{\Omega} \sup _{\boldsymbol{\xi} \in[0,1]^{n}}\left|\phi(\mathbf{x}, \boldsymbol{\xi})-\tilde{\phi}_{m}(\mathbf{x}, \boldsymbol{\xi})\right|_{\mathcal{L}}^{q} d \mathbf{x} \rightarrow 0 \quad \text { as } m \rightarrow+\infty .
\end{aligned}
$$

Using these three convergence results in 2.31) gives 2.19) and proves the proposition.

\subsection{Two-Scale Convergence criterion}

Once the Injection Lemma gotten, the following Theorem, which is important for Homogenization issues, may be proved relatively easily.

Theorem 2.13. If a sequence $\left(u^{\varepsilon}\right)$ is bounded in $L^{p}\left(\Omega ; \mathcal{L}^{\prime}\right)$, i.e. if

$$
\left\|u^{\varepsilon}\right\|_{L^{p}\left(\Omega ; \mathcal{L}^{\prime}\right)}=\left(\int_{\Omega}\left|u^{\varepsilon}(\mathbf{x})\right|_{\mathcal{L}^{\prime}}^{p} d \mathbf{x}\right)^{\frac{1}{p}} \leq c
$$

for a constant $c$ independent of $\varepsilon$, then, there exists a profile $U \in L^{p}\left(\Omega ; L_{\#}^{p}\left(\mathbb{R}^{n} ; \mathcal{L}^{\prime}\right)\right)$ such that, up to a subsequence,

$\left(u^{\varepsilon}\right)$ Two-Scale converges to $U$.

Proof. The proof of this theorem essentially uses two ingredients: the sequential Banach-Alaoglu Theorem and the Riesz Representation Theorem. 
In a first stage of the proof, the Injection Lemma and assumption 2.35 is used to get that, for any function $\phi=\phi(\mathbf{x}, \boldsymbol{\xi}) \in L^{q}\left(\Omega ; \mathcal{C}_{\#}^{0}\left(\mathbb{R}^{n} ; \mathcal{L}\right)\right)((1 / p)+(1 / q)=1)$,

$$
\begin{aligned}
\left|\int_{\Omega} \mathcal{L}^{\prime}\left\langle u^{\varepsilon}(\mathbf{x}), \phi\left(\mathbf{x}, \frac{\mathbf{x}}{\varepsilon}\right)\right\rangle_{\mathcal{L}} d \mathbf{x}\right| & \leq c\left\|[\phi]^{\varepsilon}\right\|_{L^{q}(\Omega, \mathcal{L})} \\
& \leq c\|\phi\|_{L^{q}\left(\Omega ; \mathcal{C}_{\#}^{0}\left(\mathbb{R}^{n} ; \mathcal{L}\right)\right)} .
\end{aligned}
$$

Hence the sequence $\left(\mu^{\varepsilon}\right)$ of applications, where

$$
\begin{aligned}
\mu^{\varepsilon}: L^{q}\left(\Omega ; \mathcal{C}_{\#}^{0}\left(\mathbb{R}^{n} ; \mathcal{L}\right)\right) & \rightarrow \quad \\
\phi & \mapsto \int_{\Omega} \mathcal{L}^{\prime}\left\langle u^{\varepsilon}(\mathbf{x}), \phi\left(\mathbf{x}, \frac{\mathbf{x}}{\varepsilon}\right)\right\rangle_{\mathcal{L}} d \mathbf{x}
\end{aligned}
$$

is bounded in the dual $\left(L^{q}\left(\Omega ; \mathcal{C}_{\#}^{0}\left(\mathbb{R}^{n} ; \mathcal{L}\right)\right)\right)^{\prime}$ of $L^{q}\left(\Omega ; \mathcal{C}_{\#}^{0}\left(\mathbb{R}^{n} ; \mathcal{L}\right)\right)$ which is a separable space.

Remark 2.14. The norm on $\left(L^{q}\left(\Omega ; \mathcal{C}_{\#}^{0}\left(\mathbb{R}^{n} ; \mathcal{L}\right)\right)\right)^{\prime}$ is

$$
\|\mu\|=\sup _{0 \neq \phi \in L^{q}\left(\Omega ; \mathcal{C}_{\#}^{0}\left(\mathbb{R}^{n} ; \mathcal{L}\right)\right)} \frac{|\langle\mu, \phi\rangle|}{\|\phi\|_{L^{q}\left(\Omega ; \mathcal{C}_{\#}^{0}\left(\mathbb{R}^{n} ; \mathcal{L}\right)\right)}} .
$$

Since $\left(\mu^{\varepsilon}\right)$ is the dual of a separable space, extracting a subsequence, there exists an application $\mu \in\left(L^{q}\left(\Omega ; \mathcal{C}_{\#}^{0}\left(\mathbb{R}^{n} ; \mathcal{L}\right)\right)\right)^{\prime}$

$$
\mu^{\varepsilon} \rightarrow \mu \quad \text { in }\left(L^{q}\left(\Omega ; \mathcal{C}_{\#}^{0}\left(\mathbb{R}^{n} ; \mathcal{L}\right)\right)\right)^{\prime} \text { weak-* }
$$

In particular, it implies

$$
\left\langle\mu^{\varepsilon}, \phi\right\rangle \rightarrow\langle\mu, \phi\rangle
$$

for any $\phi \in L^{q}\left(\Omega ; \mathcal{C}_{\#}^{0}\left(\mathbb{R}^{n} ; \mathcal{L}\right)\right)$.

The beginning of the second stage of the proof consists in making $\varepsilon \rightarrow 0$ in (2.37). The left hand side converges towards $\langle\mu, \phi\rangle$ and, according to Proposition 2.12, the right hand side converges towards $c\|\phi\|_{L^{q}\left(\Omega ; L_{\#}^{q}\left(\mathbb{R}^{n} ; \mathcal{L}\right)\right)}$. Hence, for every function in $\phi \in L^{q}\left(\Omega ; \mathcal{C}_{\#}^{0}\left(\mathbb{R}^{n} ; \mathcal{L}\right)\right)$,

$$
\langle\mu, \phi\rangle \leq c\|\phi\|_{L^{q}\left(\Omega ; L_{\#}^{q}\left(\mathbb{R}^{n} ; \mathcal{L}\right)\right)}
$$

Knowing that $L^{q}\left(\Omega ; \mathcal{C}_{\#}^{0}\left(\mathbb{R}^{n} ; \mathcal{L}\right)\right)$ is dense in $L^{q}\left(\Omega ; L_{\#}^{q}\left(\mathbb{R}^{n} ; \mathcal{L}\right)\right)$ - whose dual is $L^{p}\left(\Omega ; L_{\#}^{p}\left(\mathbb{R}^{n} ; \mathcal{L}^{\prime}\right)\right)$, applying the Riesz Representation Theorem, it may be deduced that there exists

$$
U \in L^{p}\left(\Omega ; L_{\#}^{p}\left(\mathbb{R}^{n} ; \mathcal{L}^{\prime}\right)\right) \text { such that }\langle\mu, \phi\rangle=\int_{\Omega} \int_{[0,1]^{n}} \mathcal{L}^{\prime}\langle U(\mathbf{x}, \boldsymbol{\xi}), \phi(\mathbf{x}, \boldsymbol{\xi})\rangle_{\mathcal{L}} d \mathbf{x} d \boldsymbol{\xi}
$$

and consequently, such that, up to a subsequence, as $\varepsilon$ goes to zero,

$$
\int_{\Omega} \mathcal{L}^{\prime}\left\langle u^{\varepsilon}(\mathbf{x}), \phi\left(\mathbf{x}, \frac{\mathbf{x}}{\varepsilon}\right)\right\rangle_{\mathcal{L}} d \mathbf{x} \rightarrow \int_{\Omega} \int_{[0,1]^{n}} \mathcal{L}^{\prime}\langle U(\mathbf{x}, \boldsymbol{\xi}), \phi(\mathbf{x}, \boldsymbol{\xi})\rangle_{\mathcal{L}} d \mathbf{x} d \boldsymbol{\xi}
$$

which is exactly 2.1 of definition 2.4 , proving 2.36 and so ending the proof. 


\subsection{Strong Two-Scale Convergence criterion}

In this section $p=q=2$ and $\mathcal{L}$ and $\mathcal{L}^{\prime}$ are the same separable Hilbert space. In order to go on gradually, I begin by the following very simple result.

Lemma 2.15. If $\psi=\psi(\mathbf{x}, \boldsymbol{\xi}) \in L^{2}\left(\Omega ; \mathcal{C}_{\#}^{0}\left(\mathbb{R}^{n} ; \mathcal{L}\right)\right)$ then the sequence of functions $\left([\psi]^{\varepsilon}\right) \subset L^{2}(\Omega$; $\left.\mathcal{C}_{\#}^{0}\left(\mathbb{R}^{n} ; \mathcal{L}\right)\right)$ defined by

$$
[\psi]^{\varepsilon}(\mathbf{x})=\psi\left(\mathbf{x}, \frac{\mathbf{x}}{\varepsilon}\right),
$$

Strongly Two-Scale converges towards $\psi$.

Proof. Applying directly 2.19) of Proposition 2.12 it can be gotten:

$$
\int_{\Omega} \mathcal{L}\left\langle\psi\left(\mathbf{x}, \frac{\mathbf{x}}{\varepsilon}\right), \phi\left(\mathbf{x}, \frac{\mathbf{x}}{\varepsilon}\right)\right\rangle_{\mathcal{L}} d \mathbf{x} \rightarrow \int_{\Omega} \int_{[0,1]^{n}} \mathcal{L}\langle\psi(\mathbf{x}, \boldsymbol{\xi}), \phi(\mathbf{x}, \boldsymbol{\xi})\rangle_{\mathcal{L}} d \mathbf{x} d \boldsymbol{\xi}
$$

for all function $\phi \in L^{2}\left(\Omega ; \mathcal{C}_{\#}^{0}\left(\mathbb{R}^{n} ; \mathcal{L}\right)\right)$, meaning that $\left([\psi]^{\varepsilon}\right)$ Two-Scale converges towards $\psi$.

Now, in view of 2.2 in Definition 2.6 since

$$
\int_{\Omega}\left|[\psi]^{\varepsilon}(\mathbf{x})-\psi\left(\mathbf{x}, \frac{\mathbf{x}}{\varepsilon}\right)\right|_{\mathcal{L}^{\prime}}^{2} d \mathbf{x} \rightarrow 0,
$$

is completely obvious, the Strong Two-Scale Convergence is insured.

As easily, the following result may also be proved.

Proposition 2.16. If $\psi$ is like in Lemma 2.15.

$$
\begin{aligned}
\left\|[\psi]^{\varepsilon}\right\|_{L^{2}(\Omega ; \mathcal{L})}=\left(\int_{\Omega} \mathcal{L}\langle\psi(\mathbf{x},\right. & \left.\left.\left.\frac{\mathbf{x}}{\varepsilon}\right), \psi\left(\mathbf{x}, \frac{\mathbf{x}}{\varepsilon}\right)\right\rangle_{\mathcal{L}} d \mathbf{x}\right)^{\frac{1}{2}} \\
& \rightarrow\left(\int_{\Omega} \int_{[0,1]^{n}} \mathcal{L}\langle\psi(\mathbf{x}, \boldsymbol{\xi}), \psi(\mathbf{x}, \boldsymbol{\xi})\rangle_{\mathcal{L}} d \mathbf{x} d \boldsymbol{\xi}\right)^{\frac{1}{2}}=\|\psi\|_{L^{2}\left(\Omega ; L_{\#}^{2}\left(\mathbb{R}^{n} ; \mathcal{L}\right)\right)}
\end{aligned}
$$

Now I will give a result, that was already evoked by Lemma 2.15. establishing the link between Strong Two-Scale Convergence and Two-Scale Convergence.

Theorem 2.17. If a sequence $\left.\left(u^{\varepsilon}\right) \subset L^{2}(\Omega ; \mathcal{L})\right)$ Strongly Two-Scale Converges towards $U$ and if $U \in L^{2}\left(\Omega ; \mathcal{C}_{\#}^{0}\left(\mathbb{R}^{n} ; \mathcal{L}\right)\right)$, then it Two-Scale Converges towards $U$.

Proof. Considering the following quantity

$$
\mathcal{I}_{\varepsilon}=\int_{\Omega} \mathcal{L}\left\langle u^{\varepsilon}(\mathbf{x})-U\left(\mathbf{x}, \frac{\mathbf{x}}{\varepsilon}\right), \phi\left(\mathbf{x}, \frac{\mathbf{x}}{\varepsilon}\right)\right\rangle_{\mathcal{L}} d \mathbf{x},
$$

for any function $\phi \in L^{2}\left(\Omega ; \mathcal{C}_{\#}^{0}\left(\mathbb{R}^{n} ; \mathcal{L}\right)\right)$, on the one hand this quantity satisfies

$$
\left|\mathcal{I}_{\varepsilon}\right| \leq\left(\int_{\Omega}\left|u^{\varepsilon}(\mathbf{x})-U\left(\mathbf{x}, \frac{\mathbf{x}}{\varepsilon}\right)\right|_{\mathcal{L}}^{2} d \mathbf{x}\right)^{\frac{1}{2}}\left(\int_{\Omega}\left|\phi\left(\mathbf{x}, \frac{\mathbf{x}}{\varepsilon}\right)\right|_{\mathcal{L}}^{2} d \mathbf{x}\right)^{\frac{1}{2}} \rightarrow 0,
$$

as $\varepsilon \rightarrow 0$, because of the Strong Two-Scale Convergence. On the other hand,

$$
\mathcal{I}_{\varepsilon}=\int_{\Omega} \mathcal{L}\left\langle u^{\varepsilon}(\mathbf{x}), \phi\left(\mathbf{x}, \frac{\mathbf{x}}{\varepsilon}\right)\right\rangle_{\mathcal{L}} d \mathbf{x}-\int_{\Omega} \mathcal{L}\left\langle U\left(\mathbf{x}, \frac{\mathbf{x}}{\varepsilon}\right), \phi\left(\mathbf{x}, \frac{\mathbf{x}}{\varepsilon}\right)\right\rangle_{\mathcal{L}} d \mathbf{x},
$$


and according to Lemma 2.15

$$
\int_{\Omega}\left\langle{ }_{\Omega}\left\langle U\left(\mathbf{x}, \frac{\mathbf{x}}{\varepsilon}\right), \phi\left(\mathbf{x}, \frac{\mathbf{x}}{\varepsilon}\right)\right\rangle_{\mathcal{L}} d \mathbf{x} \rightarrow \int_{\Omega} \int_{[0,1]^{n}} \mathcal{L}\langle U(\mathbf{x}, \boldsymbol{\xi}), \phi(\mathbf{x}, \boldsymbol{\xi})\rangle_{\mathcal{L}} d \mathbf{x} d \boldsymbol{\xi} .\right.
$$

Using 2.51 and 2.53 it is gotten that, as $\varepsilon$ goes to 0 ,

$$
\int_{\Omega} \mathcal{L}\left\langle u^{\varepsilon}(\mathbf{x}), \phi\left(\mathbf{x}, \frac{\mathbf{x}}{\varepsilon}\right)\right\rangle_{\mathcal{L}} d \mathbf{x} \rightarrow \int_{\Omega} \int_{[0,1]^{n}} \mathcal{L}\langle U(\mathbf{x}, \boldsymbol{\xi}), \phi(\mathbf{x}, \boldsymbol{\xi})\rangle_{\mathcal{L}} d \mathbf{x} d \boldsymbol{\xi}
$$

i.e. $\left(u^{\varepsilon}\right)$ Two-Scale Converges to $U$, ending the proof.

Now, I give the important Theorem concerning Strong Two-Scale Convergence.

Theorem 2.18. If a sequence $\left(u^{\varepsilon}\right) \subset L^{2}(\Omega ; \mathcal{L})$ Two-Scale converges towards a profile $U$, if $U \in$ $L^{2}\left(\Omega ; \mathcal{C}_{\#}^{0}\left(\mathbb{R}^{n} ; \mathcal{L}\right)\right)$ and if

$$
\lim _{\varepsilon \rightarrow 0}\left\|u^{\varepsilon}\right\|_{L^{2}(\Omega ; \mathcal{L})}=\|U\|_{L^{2}\left(\Omega ; L^{2}\left([0,1]^{n} ; \mathcal{L}\right)\right.}
$$

then

$$
\left(u^{\varepsilon}\right) \text { Strongly Two-Scale converges to } U \text {, }
$$

and, for any sequence $\left(v^{\varepsilon}\right) \subset L^{2}(\Omega ; \mathcal{L})$ Two-Scale converging towards a profile $V$,

$$
\mathcal{L}\left\langle u^{\varepsilon}, v^{\varepsilon}\right\rangle_{\mathcal{L}} \rightarrow \int_{[0,1]^{n}} \mathcal{\mathcal { L }}\langle U(., \boldsymbol{\xi}), V(., \boldsymbol{\xi})\rangle_{\mathcal{L}} d \boldsymbol{\xi}, \quad \text { in } \mathcal{D}^{\prime}(\Omega) .
$$

Proof. The proof of the first part of the Theorem consists just in computing:

$$
\begin{aligned}
\int_{\Omega}\left|u^{\varepsilon}(\mathbf{x})-U\left(\mathbf{x}, \frac{\mathbf{x}}{\varepsilon}\right)\right|_{\mathcal{L}}^{2} d \mathbf{x} & = \\
& \int_{\Omega}\left|u^{\varepsilon}(\mathbf{x})\right|_{\mathcal{L}}^{2} d \mathbf{x}-2 \int_{\Omega} \mathcal{L}\left\langle u^{\varepsilon}(\mathbf{x}), U\left(\mathbf{x}, \frac{\mathbf{x}}{\varepsilon}\right)\right\rangle_{\mathcal{L}} d \mathbf{x}+\int_{\Omega}\left|U\left(\mathbf{x}, \frac{\mathbf{x}}{\varepsilon}\right)\right|_{\mathcal{L}}^{2} d \mathbf{x}
\end{aligned}
$$

and in passing to the limit, as $\varepsilon$ goes to 0 , using the assumptions of the Theorem,

$$
\begin{aligned}
\lim _{\varepsilon \rightarrow 0} \int_{\Omega}\left|u^{\varepsilon}(\mathbf{x})-U\left(\mathbf{x}, \frac{\mathbf{x}}{\varepsilon}\right)\right|_{\mathcal{L}}^{2} d \mathbf{x}=\lim _{\varepsilon \rightarrow 0} \int_{\Omega}\left|u^{\varepsilon}(\mathbf{x})\right|_{\mathcal{L}}^{2} d \mathbf{x} \\
-2 \int_{\Omega} \int_{[0,1]^{n}} \mathcal{L}\langle U(\mathbf{x}, \boldsymbol{\xi}), U(\mathbf{x}, \boldsymbol{\xi})\rangle_{\mathcal{L}} d \mathbf{x} d \boldsymbol{\xi}+\int_{\Omega} \int_{[0,1]^{n}}|U(\mathbf{x}, \boldsymbol{\xi})|_{\mathcal{L}}^{2} d \mathbf{x} d \boldsymbol{\xi}=0
\end{aligned}
$$

In order to prove the second part of the Theorem, for any test function $\varphi \in \mathcal{D}(\Omega)$ the following quantity is computed:

$$
\begin{aligned}
\mathcal{D}^{\prime}\left\langle\mathcal{L}\left\langle u^{\varepsilon}, v^{\varepsilon}\right\rangle_{\mathcal{L}}, \varphi\right\rangle_{\mathcal{D}}= & \int_{\Omega} \mathcal{L}\left\langle u^{\varepsilon}(\mathbf{x}), v^{\varepsilon}(\mathbf{x})\right\rangle_{\mathcal{L}} \varphi(\mathbf{x}) d \mathbf{x}= \\
& \int_{\Omega} \mathcal{L}\left\langle U\left(\mathbf{x}, \frac{\mathbf{x}}{\varepsilon}\right), v^{\varepsilon}(\mathbf{x})\right\rangle_{\mathcal{L}} \varphi(\mathbf{x}) d \mathbf{x}-\int_{\Omega} \mathcal{L}\left\langle u^{\varepsilon}(\mathbf{x})-U\left(\mathbf{x}, \frac{\mathbf{x}}{\varepsilon}\right), v^{\varepsilon}(\mathbf{x})\right\rangle_{\mathcal{L}} \varphi(\mathbf{x}) d \mathbf{x} .
\end{aligned}
$$

Since $u^{\varepsilon}(\mathbf{x})-U\left(\mathbf{x}, \frac{\mathbf{x}}{\varepsilon}\right) \rightarrow 0$, the second term of the right hand side is such that

$$
\int_{\Omega} \mathcal{L}\left\langle u^{\varepsilon}(\mathbf{x})-U\left(\mathbf{x}, \frac{\mathbf{x}}{\varepsilon}\right), v^{\varepsilon}(\mathbf{x})\right\rangle_{\mathcal{L}} \varphi(\mathbf{x}) d \mathbf{x} \rightarrow 0
$$


as $\varepsilon$ goes to 0 . A direct calculation gives the behavior of the first term as $\varepsilon$ goes to 0 :

$$
\begin{gathered}
\int_{\Omega} \mathcal{L}\left\langle U\left(\mathbf{x}, \frac{\mathbf{x}}{\varepsilon}\right), v^{\varepsilon}(\mathbf{x})\right\rangle_{\mathcal{L}} \varphi(\mathbf{x}) d \mathbf{x}=\int_{\Omega} \mathcal{L}\left\langle v^{\varepsilon}(\mathbf{x}), U\left(\mathbf{x}, \frac{\mathbf{x}}{\varepsilon}\right)\right\rangle_{\mathcal{L}} \varphi(\mathbf{x}) d \mathbf{x}= \\
\int_{\Omega} \mathcal{L}\left\langle v^{\varepsilon}(\mathbf{x}), \varphi(\mathbf{x}) U\left(\mathbf{x}, \frac{\mathbf{x}}{\varepsilon}\right)\right\rangle_{\mathcal{L}} d \mathbf{x} \rightarrow \int_{\Omega} \int_{[0,1]^{n}} \mathcal{L}\langle V(\mathbf{x}, \boldsymbol{\xi}), \varphi(\mathbf{x}) U(\mathbf{x}, \boldsymbol{\xi})\rangle_{\mathcal{L}} d \mathbf{x} d \boldsymbol{\xi} \\
=\int_{\Omega} \int_{[0,1]^{n}} \mathcal{L}\langle V(\mathbf{x}, \boldsymbol{\xi}), U(\mathbf{x}, \boldsymbol{\xi})\rangle_{\mathcal{L}} \varphi(\mathbf{x}) d \mathbf{x} d \boldsymbol{\xi}=\int_{\Omega} \int_{[0,1]^{n}} \mathcal{L}\langle U(\mathbf{x}, \boldsymbol{\xi}), V(\mathbf{x}, \boldsymbol{\xi})\rangle_{\mathcal{L}} \varphi(\mathbf{x}) d \mathbf{x} d \boldsymbol{\xi}
\end{gathered}
$$

coupling 2.60, (2.61) and 2.62) gives

$$
\mathcal{D}^{\prime}\left\langle\mathcal{L}\left\langle u^{\varepsilon}, v^{\varepsilon}\right\rangle_{\mathcal{L}}, \varphi\right\rangle_{\mathcal{D}} \rightarrow \int_{\Omega} \int_{[0,1]^{n}} \mathcal{L}\langle U(\mathbf{x}, \boldsymbol{\xi}), V(\mathbf{x}, \boldsymbol{\xi})\rangle_{\mathcal{L}} \varphi(\mathbf{x}) d \mathbf{x} d \boldsymbol{\xi}
$$

for any test function $\varphi \in \mathcal{D}(\Omega)$, as $\varepsilon$ goes to 0 , i.e (2.57), ending the proof.

\section{Application : Homogenization of linear Singularly Perturbed HYPERBOLIC EQUATIONS}

Here, I show how to homogenize a linear Singularly Perturbed Hyperbolic Equation with a method based on Two-Scale Convergence. As said in the Introduction, this equation is related to Tokamak Plasma Physics. The setting is a very simplified one. A more general setting may be found in Frénod, Raviart \& Sonnendrücker [18] despite the presentation is different: In [18], we used TwoScale Convergence to justify Asymptotic Expansion while here the Two-Scale Convergence based method is used as a constructive Homogenization Method.

\subsection{Equation of interest and setting}

The considered equation is the following:

$$
\begin{aligned}
& \frac{\partial u^{\varepsilon}}{\partial t}+\mathbf{a} \cdot \nabla u^{\varepsilon}+\frac{1}{\varepsilon} \mathbf{b} \cdot \nabla u^{\varepsilon}=0, \\
& u_{\mid t=0}^{\varepsilon}=u_{0} .
\end{aligned}
$$

This equation (that is understood in a weak sense) is set for $u^{\varepsilon}=u^{\varepsilon}(t, \mathbf{x})$ with $\mathbf{x} \in \mathbb{R}^{d}$ and $t \in[0, T)$, for a given $T>0$. Concerning $\mathbf{a}$, it is assumed that $\mathbf{a}=\mathbf{a}(\mathbf{x})$ does not depend on time $t$, is very regular and that its divergence $\nabla \cdot \mathbf{a}$ is zero. (Those assumptions can be relaxed but it complicates calculations.) Concerning $\mathbf{b}$ the following assumptions (which can essentially not be relaxed) are done: $\mathbf{b}=\mathbf{b}(\mathbf{x})=M \mathbf{x}$, where $M$ is a matrix such that $\operatorname{tr} M=0$, and such that $\tau \mapsto e^{\tau M}$ is periodic of period 1 .

Remark 3.1. According to those assumptions, the divergence $\nabla \cdot \mathbf{b}$ of $\mathbf{b}$ is zero, and since $\mathbf{X}(\tau)=$ $e^{\tau M} \mathbf{x}$ is solution to

$$
\frac{\partial \mathbf{X}}{\partial \tau}=M \mathbf{X}(=b(\mathbf{X})), \mathbf{X}(0)=\mathbf{x},
$$

the characteristics associated with operator $(\mathbf{b} \cdot \nabla)$ are periodic of period 1 and preserve the Lebesgue measure. 


\subsection{A priori estimate}

Multiplying equation 3.1 by $u^{\varepsilon}$ (or more precisely by a regularization of $u^{\varepsilon}$ and making the regularization parameter to go to 0 ) and integrating over $\mathbb{R}^{d}$ gives

$$
\frac{d\left(\int_{\mathbb{R}^{d}}\left|u^{\varepsilon}\right|^{2} d \mathbf{x}\right)}{d t}=0
$$

since

$$
\int_{\mathbb{R}^{d}} \mathbf{a} \cdot \nabla u^{\varepsilon} u^{\varepsilon} d x=-\int_{\mathbb{R}^{d}} \mathbf{a} \cdot \nabla u^{\varepsilon} u^{\varepsilon} d \mathbf{x}-\int_{\mathbb{R}^{d}} \nabla \cdot \mathbf{a} u^{\varepsilon} u^{\varepsilon} d \mathbf{x}=-\int_{\mathbb{R}^{d}} \mathbf{a} \cdot \nabla u^{\varepsilon} u^{\varepsilon} d \mathbf{x}=0 .
$$

Integrating 3.4 from 0 to $t$ yields

$$
\int_{\mathbb{R}^{d}}\left|u^{\varepsilon}(t, .)\right|^{2} d \mathbf{x}=\int_{\mathbb{R}^{d}}\left|u_{0}\right|^{2} d \mathbf{x},
$$

and consequently

$$
\left\|u^{\varepsilon}\right\|_{L^{2}\left([0, T) ; L^{2}\left(\mathbb{R}^{d}\right)\right)}=\int_{0}^{T} \int_{\mathbb{R}^{d}}\left|u^{\varepsilon}\right|^{2} d \mathbf{x} d t=T \int_{\mathbb{R}^{d}}\left|u_{0}\right|^{2} d \mathbf{x} .
$$

As a consequence, by standard PDE theory arguments, the following result can be claimed.

Lemma 3.2. If $u_{0} \in L^{2}\left(\mathbb{R}^{d}\right)$, then the sequence $\left(u^{\varepsilon}\right)$ is bounded in $L^{2}\left([0, T) ; L^{2}\left(\mathbb{R}^{d}\right)\right)$. Hence, up to a subsequence

$$
\begin{gathered}
\left(u^{\varepsilon}\right) \text { Two-Scale Converges to } U=U(t, \tau, \mathbf{x}) \in L^{2}\left([0, T) ; L_{\#}^{2}\left(\left(\mathbb{R} ; L^{2}\left(\mathbb{R}^{d}\right)\right)\right),\right. \\
u^{\varepsilon} \rightarrow u=\int_{0}^{1} U(., \tau, .) d \tau \text { in } L^{2}\left([0, T) ; L^{2}\left(\mathbb{R}^{d}\right)\right) \text { weak. }
\end{gathered}
$$

\subsection{Weak Formulation with Oscillating Test Functions}

From any function $\phi=\phi(t, \tau, \mathbf{x}) \in \mathcal{C}^{1}\left([0, T) ; \mathcal{C}_{\#}^{1}\left(\left(\mathbb{R} ; \mathcal{C}^{1}\left(\mathbb{R}^{d}\right)\right)\right)\right.$ it is possible to define $[\phi]^{\varepsilon}$ by

$$
[\phi]^{\varepsilon}(t, \mathbf{x})=\phi\left(t, \frac{t}{\varepsilon}, \mathbf{x}\right) .
$$

Since

$$
\frac{\partial[\phi]^{\varepsilon}}{\partial t}=\left[\frac{\partial \phi}{\partial t}\right]^{\varepsilon}+\frac{1}{\varepsilon}\left[\frac{\partial \phi}{\partial \tau}\right]^{\varepsilon}
$$

multiplying $(3.1)$ by $[\phi]^{\varepsilon}$ and integrating the result by parts, the following Weak Formulation with Oscillating Test Functions is gotten:

$$
\int_{0}^{T} \int_{\mathbb{R}^{d}} u^{\varepsilon}\left(\left[\frac{\partial \phi}{\partial t}\right]^{\varepsilon}+\frac{1}{\varepsilon}\left[\frac{\partial \phi}{\partial \tau}\right]^{\varepsilon}+\mathbf{a} \cdot[\nabla \phi]^{\varepsilon}+\frac{1}{\varepsilon} \mathbf{b} \cdot[\nabla \phi]^{\varepsilon}\right) d \mathbf{x} d t+\int_{\mathbb{R}^{d}} u_{0} \phi(0,0, .) d \mathbf{x}=0 .
$$

\subsection{Order 0 Homogenization - Constraint}

Multiplying Weak Formulation with Oscillating Test Functions 3.12 by $\varepsilon$ and passing to the limit using the Two-Scale Convergence, we obtain:

$$
\int_{0}^{T} \int_{0}^{1} \int_{\mathbb{R}^{d}} U\left(\frac{\partial \phi}{\partial \tau}+\mathbf{b} \cdot \nabla \phi\right) d \mathbf{x} d \tau d t=0
$$


that is nothing but a weak formulation of

$$
\frac{\partial U}{\partial \tau}+\mathbf{b} \cdot \nabla U=0
$$

This last equation says that $U$ is constant along the characteristics of operator $(\mathbf{b} \cdot \nabla)$. Hence the following Lemma is true.

Lemma 3.3. There exists a function $V=V(t, \mathbf{y}) \in L^{2}\left([0, T) ; L^{2}\left(\mathbb{R}^{d}\right)\right)$ such that $U(t, \tau, \mathbf{x})=$ $V\left(t, e^{-\tau M} \mathbf{x}\right)$.

Remark 3.4. The result of this Lemma may also be gotten by direct computations. For instance,

$$
\begin{aligned}
\frac{\partial\left(V\left(t, e^{-\tau M} \mathbf{x}\right)\right)}{\partial \tau}+\mathbf{b} \cdot \nabla\left(V\left(t, e^{-\tau M} \mathbf{x}\right)\right) & = \\
& \left.\left.\nabla V\left(t, e^{-\tau M} \mathbf{x}\right)\right) \cdot\left(\left(-e^{-\tau M}\right) M \mathbf{x}\right)+\left(\left(e^{-\tau M}\right) M \mathbf{x}\right) \cdot \nabla V\left(t, e^{-\tau M} \mathbf{x}\right)\right)=0
\end{aligned}
$$

\subsection{Order 0 Homogenization - Equation for $V$}

From any regular function $\gamma=\gamma(t, \mathbf{y}) \in \mathcal{C}^{1}\left([0, T) ; \mathcal{C}^{1}\left(\mathbb{R}^{d}\right)\right), \phi$ defined by $\phi(t, \tau, \mathbf{x})=\gamma\left(t, e^{-\tau M} \mathbf{x}\right)$ is regular and satisfies

$$
\frac{\partial \phi}{\partial \tau}+\mathbf{b} \cdot \nabla \phi=0
$$

Using such functions in Weak Formulation with Oscillating Test Functions (3.12) cancels the terms in factor of $1 / \varepsilon$ :

$$
\int_{0}^{T} \int_{\mathbb{R}^{d}} u^{\varepsilon}\left(\left[\frac{\partial \phi}{\partial t}\right]^{\varepsilon}+\mathbf{a} \cdot[\nabla \phi]^{\varepsilon}\right) d \mathbf{x} d t+\int_{\mathbb{R}^{d}} u_{0} \phi(0,0, .) d \mathbf{x}=0 .
$$

Passing to the limit yields

$$
\int_{0}^{T} \int_{0}^{1} \int_{\mathbb{R}^{d}} U(t, \tau, \mathbf{x})\left(\frac{\partial \phi}{\partial t}(t, \tau, \mathbf{x})+\mathbf{a}(\mathbf{x}) \cdot \nabla \phi(t, \tau, \mathbf{x})\right) d \mathbf{x} d \tau d t+\int_{\mathbb{R}^{d}} u_{0} \phi(0,0, .) d \mathbf{x}=0,
$$

and using expression of $U$ in terms of $V$ and of $\phi$ in terms of $\gamma$, since

$$
\frac{\partial \phi}{\partial t}(t, \tau, \mathbf{x})=\frac{\partial \gamma}{\partial t}\left(t, e^{-\tau M} \mathbf{x}\right) \text { and } \nabla \phi(t, \tau, \mathbf{x})=\left(e^{-\tau M}\right)^{T} \nabla \gamma\left(t, e^{-\tau M} \mathbf{x}\right)
$$

gives

$$
\begin{aligned}
\int_{0}^{T} \int_{0}^{1} \int_{\mathbb{R}^{d}} V\left(t, e^{-\tau M} \mathbf{x}\right)\left(\frac{\partial \gamma}{\partial t}\left(t, e^{-\tau M} \mathbf{x}\right)+e^{-\tau M} \mathbf{a}(\mathbf{x}) \cdot \nabla \gamma\left(t, e^{-\tau M} \mathbf{x}\right)\right) d \mathbf{x} d \tau d t & \\
& +\int_{\mathbb{R}^{d}} u_{0}(\mathbf{x}) \gamma(0, \mathbf{x}) d \mathbf{x}=0
\end{aligned}
$$

In the first integral of the left hand side we make the change of variables $(t, \tau, \mathbf{x}) \mapsto\left(t, \tau, \mathbf{y}=e^{-\tau M} \mathbf{x}\right)$ which preserves the Lebesgue measure and which reverse transform is $(t, \tau, \mathbf{y}) \mapsto\left(t, \tau, \mathbf{x}=e^{\tau M} \mathbf{y}\right)$. It gives

$$
\begin{aligned}
\int_{0}^{T} \int_{0}^{1} \int_{\mathbb{R}^{d}} V(t, \mathbf{y})\left(\frac{\partial \gamma}{\partial t}(t, \mathbf{y})+e^{-\tau M} \mathbf{a}\left(e^{\tau M} \mathbf{y}\right) \cdot \nabla \gamma(t, \mathbf{y})\right) d \mathbf{y} d \tau d t & \\
& +\int_{\mathbb{R}^{d}} u_{0}(\mathbf{y}) \gamma(0, \mathbf{y}) d \mathbf{y}=0
\end{aligned}
$$


or

$$
\begin{aligned}
\int_{0}^{T} \int_{\mathbb{R}^{d}} V(t, \mathbf{y})\left(\frac{\partial \gamma}{\partial t}(t, \mathbf{y})+\left(\int_{0}^{1} e^{-\tau M} \mathbf{a}\left(e^{\tau M} \mathbf{y}\right) d \tau\right) \cdot \nabla \gamma(t, \mathbf{y})\right) d \mathbf{y} d t & \\
& +\int_{\mathbb{R}^{d}} u_{0}(\mathbf{y}) \gamma(0, \mathbf{y}) d \mathbf{y}=0
\end{aligned}
$$

Which says:

Theorem 3.5. Under assumption of Lemma 3.2. function $V(t, \mathbf{y})$ linked by Lemma 3.3 with the Two-Scale limit $U(t, \tau, \mathbf{x})$ of $\left(u^{\varepsilon}\right)$ is solution to

$$
\begin{aligned}
& \frac{\partial V}{\partial t}+\left(\int_{0}^{1} e^{-\sigma M} \mathbf{a}\left(e^{\sigma M} \mathbf{y}\right) d \sigma\right) \cdot \nabla V=0 \\
& V_{\mid t=0}=u_{0}
\end{aligned}
$$

Remark 3.6. Clearly, the solution of (3.23) and (3.24) is unique. As a consequence, the whole sequence $\left(u^{\varepsilon}\right)$ converges (Two-Scale towards $U$, and weak-* towards $u$ )

\subsection{Order 1 Homogenization - Preparations: equation for $U$ and $u$}

Because of the linearity of the problem, it is possible to deduce from (3.23) an equation for $U$ also. Indeed, since $\nabla U(t, \tau, \mathbf{x})=\left(e^{-\tau M}\right)^{T} \nabla V\left(t, e^{-\tau M} \mathbf{x}\right)$ or $\nabla V\left(t, e^{-\tau M} \mathbf{x}\right)=\left(e^{\tau M}\right)^{T} \nabla U(t, \tau, \mathbf{x})$, writing 3.23 in $\mathbf{y}=e^{-\tau M} \mathbf{x}$, we obtain that

$$
\begin{aligned}
& 0=\frac{\partial\left(V\left(t, e^{-\tau M} \mathbf{x}\right)\right)}{\partial t}+\left(\int_{0}^{1} e^{-\sigma M} \mathbf{a}\left(e^{\sigma M} e^{-\tau M} \mathbf{x}\right) d \sigma\right) \cdot \nabla V\left(t, e^{-\tau M} \mathbf{x}\right) \\
&=\frac{\partial U}{\partial t}+\left(e^{\tau M} \int_{0}^{1} e^{-\sigma M} \mathbf{a}\left(e^{(\sigma-\tau) M} \mathbf{x}\right) d \sigma\right) \cdot \nabla U=\frac{\partial U}{\partial t}+\left(\int_{0}^{1} e^{(\tau-\sigma) M} \mathbf{a}\left(e^{(\sigma-\tau) M} \mathbf{x}\right) d \sigma\right) \cdot \nabla U \\
&=\frac{\partial U}{\partial t}+\left(\int_{0}^{1} e^{-\sigma M} \mathbf{a}\left(e^{\sigma M} \mathbf{x}\right) d \sigma\right) \cdot \nabla U
\end{aligned}
$$

the last equality being gotten from periodicity of $\sigma \mapsto e^{\sigma M}$.

Now, since $\left(\int_{0}^{1} e^{-\sigma M} \mathbf{a}\left(e^{(\sigma) M} \mathbf{x}\right) d \sigma\right)$ does not depend on $\tau$ and because of 3.9 , integrating 3.25 , gives

$$
\frac{\partial u}{\partial t}+\left(\int_{0}^{1} e^{-\sigma M} \mathbf{a}\left(e^{\sigma M} \mathbf{x}\right) d \sigma\right) \cdot \nabla u=0
$$

Finally, since $u(0, \mathbf{x})=\int_{0}^{1} U(0, \tau, \mathbf{x}) d \tau$ and $U(0, \tau, \mathbf{x})=V\left(0, e^{-\tau M} \mathbf{x}\right)=u_{0}\left(e^{-\tau M} \mathbf{x}\right)$, the following Lemma is true

Lemma 3.7. Under assumption of Lemma 3.2, the Two-Scale limit $U(t, \tau, \mathbf{x})$ of $\left(u^{\varepsilon}\right)$ and its weak-* limit $u$ are solutions to

$$
\begin{aligned}
& \frac{\partial U}{\partial t}+\left(\int_{0}^{1} e^{-\sigma M} \mathbf{a}\left(e^{\sigma M} \mathbf{x}\right) d \sigma\right) \cdot \nabla U=0 \\
& U_{\mid t=0}=u_{0}\left(e^{-\tau M} \mathbf{x}\right)
\end{aligned}
$$


and

$$
\begin{aligned}
& \frac{\partial u}{\partial t}+\left(\int_{0}^{1} e^{-\sigma M} \mathbf{a}\left(e^{\sigma M} \mathbf{x}\right) d \sigma\right) \cdot \nabla u=0 \\
& u_{\mid t=0}=\int_{0}^{1} u_{0}\left(e^{-\tau M} \mathbf{x}\right) d \sigma .
\end{aligned}
$$

\subsection{Order 1 Homogenization - Strong Two-Scale convergence of $U$}

Because

$$
\frac{\partial\left(u^{\varepsilon}\right)^{2}}{\partial t}=2 u^{\varepsilon} \frac{\partial u^{\varepsilon}}{\partial t} \quad \text { and } \quad \nabla\left(u^{\varepsilon}\right)^{2}=2 u^{\varepsilon} \nabla u^{\varepsilon},
$$

multiplying (3.1) by $2 u^{\varepsilon}$, we obtain that $\left(u^{\varepsilon}\right)^{2}$ is solution to:

$$
\begin{aligned}
& \frac{\partial\left(u^{\varepsilon}\right)^{2}}{\partial t}+\mathbf{a} \cdot \nabla\left(u^{\varepsilon}\right)^{2}+\frac{1}{\varepsilon} \mathbf{b} \cdot \nabla\left(u^{\varepsilon}\right)^{2}=0, \\
& \left(u^{\varepsilon}\right)_{\mid t=0}^{2}=u_{0}^{2} .
\end{aligned}
$$

Hence if $u_{0}^{2}$ is in $L^{2}\left(\mathbb{R}^{d}\right)$, i.e. if $u_{0} \in L^{4}\left(\mathbb{R}^{d}\right)$, it is possible to do the same for equation 3.32 as for (3.1) and find that $\left(u^{\varepsilon}\right)^{2}$ Two-Scale converges to a profile, called $Z$, and that $Z$ is solution to

$$
\begin{aligned}
& \frac{\partial Z}{\partial t}+\left(\int_{0}^{1} e^{-\sigma M} \mathbf{a}\left(e^{\sigma M} \mathbf{x}\right) d \sigma\right) \cdot \nabla Z=0, \\
& Z_{\mid t=0}=u_{0}^{2}\left(e^{-\tau M} \mathbf{x}\right)
\end{aligned}
$$

leading to the conclusion that $Z=U^{2}$ or

$$
\left(\left(u^{\varepsilon}\right)^{2}\right) \text { Two-Scale Converges to } U^{2} \text {. }
$$

From (3.36), it is easy to get that

$$
\left\|u^{\varepsilon}\right\|_{L^{2}\left([0, T) ; L^{2}\left(\mathbb{R}^{d}\right)\right)} \rightarrow\|U\|_{L^{2}\left([0, T) ; L_{\#}^{2}\left(\left(\mathbb{R} ; L^{2}\left(\mathbb{R}^{d}\right)\right)\right)\right.},
$$

as $\varepsilon \rightarrow 0$.

Indeed, we only need to consider for any $\delta>0$ the regular function $\beta^{\delta}=\beta^{\delta}(\mathbf{x})$ which is such that $\beta^{\delta}(\mathbf{x})=1$ when $|\mathbf{x}|<1 / \delta, \beta^{\delta}(\mathbf{x})=0$ when $|\mathbf{x}|>1 / \delta+1$ and $0 \leq \beta^{\delta} \leq 1$. Clearly from (3.36), for any $\delta$,

$$
\int_{0}^{T} \int_{\mathbb{R}^{d}}\left(u^{\varepsilon}\right)^{2} \beta^{\delta} d \mathbf{x} d t \rightarrow \int_{0}^{T} \int_{0}^{1} \int_{\mathbb{R}^{d}} U^{2} \beta^{\delta} d \mathbf{x} d \tau d t
$$

and as $\delta \rightarrow 0$,

$$
\begin{gathered}
\int_{0}^{T} \int_{\mathbb{R}^{d}}\left(u^{\varepsilon}\right)^{2} \beta^{\delta} d \mathbf{x} d t \rightarrow\left\|u^{\varepsilon}\right\|_{L^{2}\left([0, T) ; L^{2}\left(\mathbb{R}^{d}\right)\right)}, \\
\int_{0}^{T} \int_{\mathbb{R}^{d}} U^{2} \beta^{\delta} d \mathbf{x} d \tau d t \rightarrow\|U\|_{L^{2}\left([0, T) ; L_{\#}^{2}\left(\left(\mathbb{R} ; L^{2}\left(\mathbb{R}^{d}\right)\right)\right)\right.} .
\end{gathered}
$$

Moreover, if $u_{0}$ is in $\mathcal{C}^{0}\left(\mathbb{R}^{d}\right)$ then, $u^{\varepsilon} \in \mathcal{C}^{0}\left([0, T) ; \mathcal{C}^{0}\left(\mathbb{R}^{d}\right)\right), U \in \mathcal{C}^{0}\left([0, T) ; \mathcal{C}_{\#}^{0}\left(\left(\mathbb{R} ; \mathcal{C}^{0}\left(\mathbb{R}^{d}\right)\right)\right)\right.$ and $V \in \mathcal{C}^{0}\left([0, T) ; \mathcal{C}^{0}\left(\mathbb{R}^{d}\right)\right)$. This can be directly deduced from the equations satisfied by those functions. 
Hence Theorem 2.18 can be invoked to deduce the next Lemma.

Lemma 3.8. If $u_{0} \in\left(L^{2} \cap L^{4} \cap \mathcal{C}^{0}\right)\left(\mathbb{R}^{d}\right)$, then in addition to every already stated results,

$$
\left(u^{\varepsilon}\right) \text { Strongly Two-Scale Converges to U. }
$$

Having this result, we know that $\left(u^{\varepsilon}-[U]^{\varepsilon}\right) \rightarrow 0$, we now can show more: $\left(\left(u^{\varepsilon}-[U]^{\varepsilon}\right) / \varepsilon\right)$ Two-Scale Converges.

\subsection{Order 1 Homogenization - Function $W_{1}$}

In a first stage, from equation 3.1, 3.14 and 3.27) we deduce

$$
\begin{aligned}
& \frac{\partial\left(u^{\varepsilon}-[U]^{\varepsilon}\right)}{\partial t}+\mathbf{a} \cdot \nabla\left(u^{\varepsilon}-[U]^{\varepsilon}\right)+\frac{1}{\varepsilon} \mathbf{b} \cdot \nabla\left(u^{\varepsilon}-[U]^{\varepsilon}\right) \\
& =-\left(\mathbf{a}-\int_{0}^{1} e^{-\sigma M} \mathbf{a}\left(e^{\sigma M} \mathbf{x}\right) d \sigma\right) \cdot \nabla[U]^{\varepsilon}, \\
& \left(u^{\varepsilon}-[U]^{\varepsilon}\right)_{\mid t=0}=0 .
\end{aligned}
$$

Multiplying this equation by $1 / \varepsilon$ we obtain

$$
\begin{aligned}
& \begin{aligned}
\frac{\partial\left(\frac{u^{\varepsilon}-[U]^{\varepsilon}}{\varepsilon}\right)}{\partial t}+\mathbf{a} \cdot \nabla\left(\frac{u^{\varepsilon}-[U]^{\varepsilon}}{\varepsilon}\right)+\frac{1}{\varepsilon} \mathbf{b} \cdot \nabla\left(\frac{u^{\varepsilon}-[U]^{\varepsilon}}{\varepsilon}\right) \\
=-\frac{1}{\varepsilon}\left(\mathbf{a}-\int_{0}^{1} e^{-\sigma M} \mathbf{a}\left(e^{\sigma M} \mathbf{x}\right) d \sigma\right) \cdot \nabla[U]^{\varepsilon}
\end{aligned} \\
& \left(\frac{u^{\varepsilon}-[U]^{\varepsilon}}{\varepsilon}\right)_{\mid t=0}=0 .
\end{aligned}
$$

The left hand side of this equation is the same as in (3.1) but the right hand side is in factor of $1 / \varepsilon$.

Hence, in a second stage, we introduce a function $W_{1}=W_{1}(t, \tau, \mathbf{y})$ such that

$$
\tilde{W}_{1}=\tilde{W}_{1}(t, \tau, \mathbf{x})=W_{1}\left(t, \tau, e^{-\tau M} \mathbf{x}\right),
$$

satisfies

$$
\frac{\partial \tilde{W}_{1}}{\partial \tau}+\mathbf{b} \cdot \nabla \tilde{W}_{1}=-\left(\mathbf{a}-\int_{0}^{1} e^{-\sigma M} \mathbf{a}\left(e^{\sigma M} \mathbf{x}\right) d \sigma\right) \cdot \nabla U
$$

Because of 3.47 , considering $\left[\tilde{W}_{1}\right]^{\varepsilon}=\left[\tilde{W}_{1}\right]^{\varepsilon}(t, \mathbf{x})=\tilde{W}_{1}(t, t / \varepsilon, \mathbf{x})$,

$$
\begin{aligned}
\frac{\partial\left[\tilde{W}_{1}\right]^{\varepsilon}}{\partial t}+\mathbf{a} \cdot \nabla\left[\tilde{W}_{1}\right]^{\varepsilon} & +\frac{1}{\varepsilon} \mathbf{b} \cdot \nabla\left[\tilde{W}_{1}\right]^{\varepsilon} \\
& =\left[\frac{\partial \tilde{W}_{1}}{\partial t}\right]^{\varepsilon}+\frac{1}{\varepsilon}\left[\frac{\partial \tilde{W}_{1}}{\partial \tau}\right]^{\varepsilon}+\mathbf{a} \cdot \nabla\left[\tilde{W}_{1}\right]^{\varepsilon}+\frac{1}{\varepsilon} \mathbf{b} \cdot \nabla\left[\tilde{W}_{1}\right]^{\varepsilon} \\
& =\left[\frac{\partial \tilde{W}_{1}}{\partial t}\right]^{\varepsilon}+\mathbf{a} \cdot \nabla\left[\tilde{W}_{1}\right]^{\varepsilon}-\frac{1}{\varepsilon}\left(\mathbf{a}-\int_{0}^{1} e^{-\sigma M} \mathbf{a}\left(e^{\sigma M} \mathbf{x}\right) d \sigma\right) \cdot \nabla[U]^{\varepsilon}
\end{aligned}
$$


Subtracting 3.48 from 3.42) gives

$$
\begin{gathered}
\frac{\partial\left(\frac{u^{\varepsilon}-[U]^{\varepsilon}}{\varepsilon}-\left[\tilde{W}_{1}\right]^{\varepsilon}\right)}{\partial t}+\mathbf{a} \cdot \nabla\left(\frac{u^{\varepsilon}-[U]^{\varepsilon}}{\varepsilon}-\left[\tilde{W}_{1}\right]^{\varepsilon}\right)+\frac{1}{\varepsilon} \mathbf{b} \cdot \nabla\left(\frac{u^{\varepsilon}-[U]^{\varepsilon}}{\varepsilon}-\left[\tilde{W}_{1}\right]^{\varepsilon}\right) \\
=-\left[\frac{\partial \tilde{W}_{1}}{\partial t}\right]^{\varepsilon}-\mathbf{a} \cdot \nabla\left[\tilde{W}_{1}\right]^{\varepsilon} \\
\left(\frac{u^{\varepsilon}-[U]^{\varepsilon}}{\varepsilon}-\left[\tilde{W}_{1}\right]^{\varepsilon}\right)_{\mid t=0}=-\left[\tilde{W}_{1}\right]_{\mid t=0}^{\varepsilon} .
\end{gathered}
$$

The goal of the third stage is to give an expression of the function $W_{1}$ : Function $\tilde{W}_{1}$ is solution of 3.47) if and only if $W_{1}$ is solution to

$$
\frac{\partial W_{1}}{\partial \tau}=-\left(\mathbf{a}\left(e^{\tau M} \mathbf{y}\right)-\int_{0}^{1} e^{-\sigma M} \mathbf{a}\left(e^{(\sigma+\tau) M} \mathbf{y}\right) d \sigma\right) \cdot \nabla U\left(t, \tau, e^{\tau M} \mathbf{y}\right)
$$

Beside this, $\nabla U\left(t, \tau, e^{\tau M} \mathbf{y}\right)=\left(e^{-\tau M}\right)^{T} \nabla\left(U\left(t, \tau, e^{\tau M} \mathbf{y}\right)\right)=\left(e^{-\tau M}\right)^{T} \nabla V(t, \mathbf{y})$, hence $W_{1}$ is solution to

$$
\begin{aligned}
\frac{\partial W_{1}}{\partial \tau}=-\left(e^{-\tau M} \mathbf{a}\left(e^{\tau M} \mathbf{y}\right)-\int_{0}^{1} e^{-(\sigma+\tau) M} \mathbf{a}\left(e^{(\sigma+\tau) M} \mathbf{y}\right) d \sigma\right) \cdot \nabla V(t, \mathbf{y}) \\
=-\left(e^{-\tau M} \mathbf{a}\left(e^{\tau M} \mathbf{y}\right)-\int_{0}^{1} e^{-\sigma M} \mathbf{a}\left(e^{\sigma M} \mathbf{y}\right) d \sigma\right) \cdot \nabla V(t, \mathbf{y})
\end{aligned}
$$

(using once again periodicity of $\tau \mapsto e^{\tau M}$ ) which is:

$$
W_{1}(t, \tau, \mathbf{y})=-\left(\int_{0}^{\tau} e^{-\sigma M} \mathbf{a}\left(e^{\sigma M} \mathbf{y}\right) d \sigma-\tau \int_{0}^{1} e^{-\sigma M} \mathbf{a}\left(e^{\sigma M} \mathbf{y}\right) d \sigma\right) \cdot \nabla V(t, \mathbf{y}) .
$$

This allows us to compute $\left[\tilde{W}_{1}\right]^{\varepsilon}$. In particular in $3.50,\left[\tilde{W}_{1}\right]_{\mid t=0}^{\varepsilon}=0$ and if $u_{0}$ is regular (for instance in $\left.\mathcal{C}^{2}\left(\mathbb{R}^{2}\right)\right)$ in addition to assumptions of Lemma 3.8. because of equation 3.27 $V$ satisfies, it is easily gotten that

$$
\left\|-\left[\frac{\partial \tilde{W}_{1}}{\partial t}\right]^{\varepsilon}-\mathbf{a} \cdot \nabla\left[\tilde{W}_{1}\right]^{\varepsilon}\right\|_{L^{\infty}\left([0, T) ; L^{2}\left(\mathbb{R}^{d}\right)\right)} \leq C_{1},
$$

for a constant $C_{1}$ not depending on $\varepsilon$.

\subsection{Order 1 Homogenization - A priori estimate and convergence}

Multiplying 3.49 by $\left(\left(u^{\varepsilon}-[U]^{\varepsilon}\right) / \varepsilon-\left[\tilde{W}_{1}\right]^{\varepsilon}\right)$, we get

$$
\frac{d\left(\int_{\mathbb{R}^{d}}\left|\frac{u^{\varepsilon}-[U]^{\varepsilon}}{\varepsilon}-\left[\tilde{W}_{1}\right]^{\varepsilon}\right|^{2} d \mathbf{x}\right)}{d t} \leq C_{1}\left(\int_{\mathbb{R}^{d}}\left|\frac{u^{\varepsilon}-[U]^{\varepsilon}}{\varepsilon}-\left[\tilde{W}_{1}\right]^{\varepsilon}\right|^{2} d \mathbf{x}\right)^{\frac{1}{2}}
$$

from which an estimate can be gotten and expressed in the following Lemma. 
Lemma 3.9. If $u_{0} \in\left(L^{2} \cap L^{4} \cap \mathcal{C}^{2}\right)\left(\mathbb{R}^{d}\right)$, then in addition to every already stated results, the sequences

$$
\frac{u^{\varepsilon}-[U]^{\varepsilon}}{\varepsilon}-\left[\tilde{W}_{1}\right]^{\varepsilon}, \text { and consequently } \frac{u^{\varepsilon}-[U]^{\varepsilon}}{\varepsilon},
$$

are bounded in $L^{2}\left([0, T) ; L^{2}\left(\mathbb{R}^{d}\right)\right)$. Then, up to subsequences,

$$
\begin{gathered}
\left(\frac{u^{\varepsilon}-[U]^{\varepsilon}}{\varepsilon}\right) \text { Two-Scale Converges to } U_{1}=U_{1}(t, \tau, \mathbf{x}) \in L^{2}\left([0, T) ; L_{\#}^{2}\left(\left(\mathbb{R} ; L^{2}\left(\mathbb{R}^{d}\right)\right)\right),\right. \\
\left(\frac{u^{\varepsilon}-[U]^{\varepsilon}}{\varepsilon}-\left[\tilde{W}_{1}\right]^{\varepsilon}\right) \text { Two-Scale Converges to } U_{1}-\tilde{W}_{1},
\end{gathered}
$$

where $W_{1}$ is defined in 3.53) and $\tilde{W}_{1}$ by 3.46).

\subsection{Order 1 Homogenization - Constraint}

For any Oscillating Test Function $\phi=\phi(t, \tau, \mathbf{x}) \in \mathcal{C}^{1}\left([0, T) ; \mathcal{C}_{\#}^{1}\left(\left(\mathbb{R} ; \mathcal{C}^{1}\left(\mathbb{R}^{d}\right)\right)\right)\right.$, it is possible to write the following Weak Formulation:

$$
\begin{aligned}
\int_{0}^{T} \int_{\mathbb{R}^{d}}\left(\frac{u^{\varepsilon}-[U]^{\varepsilon}}{\varepsilon}-\left[\tilde{W}_{1}\right]^{\varepsilon}\right)\left(\left[\frac{\partial \phi}{\partial t}\right]^{\varepsilon}+\right. & \left.\frac{1}{\varepsilon}\left[\frac{\partial \phi}{\partial \tau}\right]^{\varepsilon}+\mathbf{a} \cdot[\nabla \phi]^{\varepsilon}+\frac{1}{\varepsilon} \mathbf{b} \cdot[\nabla \phi]^{\varepsilon}\right) d \mathbf{x} d t \\
& =\int_{0}^{T} \int_{\mathbb{R}^{d}}\left(-\left[\frac{\partial \tilde{W}_{1}}{\partial t}\right]^{\varepsilon}-\mathbf{a} \cdot \nabla\left[\tilde{W}_{1}\right]^{\varepsilon}\right)[\phi]^{\varepsilon} d \mathbf{x} d t
\end{aligned}
$$

Multiplying this equation by $\varepsilon$ and passing to the limit yields the next constrain equation:

$$
\frac{\partial\left(U_{1}-\tilde{W}_{1}\right)}{\partial \tau}+\mathbf{b} \cdot \nabla\left(U_{1}-\tilde{W}_{1}\right)=0
$$

Hence the following Lemma is true.

Lemma 3.10. There exists a function $V_{1}=V_{1}(t, \mathbf{y}) \in L^{2}\left([0, T) ; L^{2}\left(\mathbb{R}^{d}\right)\right)$ such that $U_{1}(t, \tau, \mathbf{x})-$ $\tilde{W}_{1}(t, \tau, \mathbf{x})=V_{1}\left(t, e^{-\tau M} \mathbf{x}\right)$ or, in other words, such that

$$
U_{1}(t, \tau, \mathbf{x})=V_{1}\left(t, e^{-\tau M} \mathbf{x}\right)+W_{1}\left(t, \tau, e^{-\tau M} \mathbf{x}\right),
$$

where $W_{1}$ is defined in 3.53.

\subsection{Order 1 Homogenization - Equation for $V_{1}$}

Using now in 3.59 Oscillating Test Function $\phi(t, \tau, \mathbf{x})=\gamma\left(t, e^{-\tau M} \mathbf{x}\right)$ for any regular function $\gamma=\gamma(t, \mathbf{y})$, the terms in factor of $\varepsilon$ cancel and passing to the limit, it gives

$$
\begin{aligned}
\int_{0}^{T} \int_{0}^{1} \int_{\mathbb{R}^{d}} V_{1}\left(t, e^{-\tau M} \mathbf{x}\right)\left(\frac{\partial \gamma}{\partial t}\right. & \left.\left(t, e^{-\tau M} \mathbf{x}\right)+e^{-\tau M} \mathbf{a}(\mathbf{x}) \cdot \nabla \gamma\left(t, e^{-\tau M} \mathbf{x}\right)\right) d \mathbf{x} d \tau d t \\
& =\int_{0}^{T} \int_{0}^{1} \int_{\mathbb{R}^{d}}\left(-\frac{\partial \tilde{W}_{1}}{\partial t}-\mathbf{a}(\mathbf{x}) \cdot \nabla \tilde{W}_{1}\right) \gamma\left(t, e^{-\tau M} \mathbf{x}\right) d \mathbf{x} d \tau d t .
\end{aligned}
$$


Making in 3.62 the change of variables $(t, \tau, \mathbf{x}) \mapsto\left(t, \tau, \mathbf{y}=e^{-\tau M} \mathbf{x}\right)$ gives

$$
\begin{aligned}
& \int_{0}^{T} \int_{0}^{1} \int_{\mathbb{R}^{d}} V_{1}(t, \mathbf{y})\left(\frac{\partial \gamma}{\partial t}(\right.\left.t, \mathbf{y})+e^{-\tau M} \mathbf{a}\left(e^{\tau M} \mathbf{y}\right) \cdot \nabla \gamma(t, \mathbf{y})\right) d \mathbf{y} d \tau d t \\
&=\int_{0}^{T} \int_{0}^{1} \int_{\mathbb{R}^{d}}\left(-\frac{\partial W_{1}}{\partial t}-e^{-\tau M} \mathbf{a}\left(e^{\tau M} \mathbf{y}\right) \cdot \nabla W_{1}\right) \gamma(t, \mathbf{y}) d \mathbf{y} d \tau d t
\end{aligned}
$$

which is the weak formulation of

$$
\begin{aligned}
& \frac{\partial V_{1}}{\partial t}+\left(\int_{0}^{1} e^{-\sigma M} \mathbf{a}\left(e^{\sigma M} \mathbf{y}\right) d \sigma\right) \cdot \nabla V_{1}=\int_{0}^{1}\left(-\frac{\partial W_{1}}{\partial t}-e^{-\tau M} \mathbf{a}\left(e^{\tau M} \mathbf{y}\right) \cdot \nabla W_{1}\right) d \tau, \\
& V_{1 \mid t=0}=0 .
\end{aligned}
$$

Now, it remains to express the right hand side of (3.64) using expression (3.53) of $W_{1}$. For this we need to compute the time derivative of $\nabla V$ and the Jacobian matrices of $-\left(\int_{0}^{\prime} e^{-\sigma M} \mathbf{a}\left(e^{\sigma M} \mathbf{y}\right) d \sigma-\right.$ $\left.\tau \int_{0}^{1} e^{-\sigma M} \mathbf{a}\left(e^{\sigma M} \mathbf{y}\right) d \sigma\right)$ and of $\nabla V$ (i.e. the Hessian matrix of $V$ ).

First, using the equation (3.23) satisfied by $V$,

$$
\begin{aligned}
& \frac{\partial(\nabla V)}{\partial t}= \\
& \quad \nabla \frac{\partial V}{\partial t}=-\left[\nabla\left[\int_{0}^{1} e^{-\sigma M} \mathbf{a}\left(e^{\sigma M} \mathbf{y}\right) d \sigma\right]\right]^{T}(\nabla V)-[\nabla \nabla V]\left(\int_{0}^{1} e^{-\sigma M} \mathbf{a}\left(e^{\sigma M} \mathbf{y}\right) d \sigma\right) .
\end{aligned}
$$

Hence,

$$
\begin{aligned}
\int_{0}^{1}-\frac{\partial W_{1}}{\partial t} d \tau= & \int_{0}^{1}-\left(\int_{0}^{\tau} e^{-\sigma M} \mathbf{a}\left(e^{\sigma M} \mathbf{y}\right) d \sigma-\tau \int_{0}^{1} e^{-\sigma M} \mathbf{a}\left(e^{\sigma M} \mathbf{y}\right) d \sigma\right) \\
& \left(\left[\nabla\left[\int_{0}^{1} e^{-\sigma M} \mathbf{a}\left(e^{\sigma M} \mathbf{y}\right) d \sigma\right]\right]^{T} \cdot(\nabla V)\right) d \tau \\
& +\int_{0}^{1}-\left(\int_{0}^{\tau} e^{-\sigma M} \mathbf{a}\left(e^{\sigma M} \mathbf{y}\right) d \sigma-\tau \int_{0}^{1} e^{-\sigma M} \mathbf{a}\left(e^{\sigma M} \mathbf{y}\right) d \sigma\right) \cdot \\
= & \left([\nabla \nabla V]\left(\int_{0}^{1} e^{-\sigma M} \mathbf{a}\left(e^{\sigma M} \mathbf{y}\right) d \sigma\right)\right) d \tau \\
+ & \left.\left.\int_{0}^{1}-\left([\nabla \nabla V]\left(\int_{0}^{1} e^{-\sigma M} \mathbf{a}\left(e^{\sigma M} \mathbf{y}\right) d \sigma\right]\right] \mathbf{a}\left(e^{\sigma M} \mathbf{y}\right) d \sigma-\tau \int_{0}^{1} e^{-\sigma M} \mathbf{a}\left(e^{\sigma M} \mathbf{y}\right) d \sigma\right)\right) . \\
& \left.\left(\int_{0}^{\tau} e^{-\sigma M} \mathbf{a}\left(e^{\sigma M} \mathbf{y}\right) d \sigma-\tau \int_{0}^{1} e^{-\sigma M} \mathbf{a}\left(e^{\sigma M} \mathbf{y}\right) d \sigma\right)\right) \cdot(\nabla V) d \tau \\
& \left(\int_{0}^{1} e^{-\sigma M} \mathbf{a}\left(e^{\sigma M} \mathbf{y}\right) d \sigma\right) d \tau .
\end{aligned}
$$


On another hand,

$$
\begin{aligned}
\int_{0}^{1} & -\left(e^{-\tau M} \mathbf{a}\left(e^{\tau M} \mathbf{y}\right)\right) \cdot \nabla W_{1} d \tau= \\
& \int_{0}^{1}\left(e^{-\tau M} \mathbf{a}\left(e^{\tau M} \mathbf{y}\right)\right) \cdot\left(\left[\nabla\left[\int_{0}^{\tau} e^{-\sigma M} \mathbf{a}\left(e^{\sigma M} \mathbf{y}\right) d \sigma-\tau \int_{0}^{1} e^{-\sigma M} \mathbf{a}\left(e^{\sigma M} \mathbf{y}\right) d \sigma\right]\right]^{T}(\nabla V)\right) d \tau \\
& +\int_{0}^{1}\left(e^{-\tau M} \mathbf{a}\left(e^{\tau M} \mathbf{y}\right)\right) \cdot\left([\nabla \nabla V]\left(\int_{0}^{\tau} e^{-\sigma M} \mathbf{a}\left(e^{\sigma M} \mathbf{y}\right) d \sigma-\tau \int_{0}^{1} e^{-\sigma M} \mathbf{a}\left(e^{\sigma M} \mathbf{y}\right) d \sigma\right)\right)= \\
& \int_{0}^{1}\left(\left[\nabla\left[\int_{0}^{\tau} e^{-\sigma M} \mathbf{a}\left(e^{\sigma M} \mathbf{y}\right) d \sigma-\tau \int_{0}^{1} e^{-\sigma M} \mathbf{a}\left(e^{\sigma M} \mathbf{y}\right) d \sigma\right]\right]\left(e^{-\tau M} \mathbf{a}\left(e^{\tau M} \mathbf{y}\right)\right)\right) \cdot(\nabla V) d \tau \\
+ & \int_{0}^{1}\left([\nabla \nabla V]\left(\int_{0}^{\tau} e^{-\sigma M} \mathbf{a}\left(e^{\sigma M} \mathbf{y}\right) d \sigma-\tau \int_{0}^{1} e^{-\sigma M} \mathbf{a}\left(e^{\sigma M} \mathbf{y}\right) d \sigma\right)\right) \cdot\left(e^{-\tau M} \mathbf{a}\left(e^{\tau M} \mathbf{y}\right)\right) d \tau .
\end{aligned}
$$

As a consequence, the right hand side of 3.64 expresses as

$$
\begin{aligned}
& \int_{0}^{1}\left(-\frac{\partial W_{1}}{\partial t}-\left(e^{-\tau M} \mathbf{a}\left(e^{\tau M} \mathbf{y}\right)\right) \cdot \nabla W_{1}\right) d \tau= \\
& \quad \int_{0}^{1}\left(\left[\nabla\left[\int_{0}^{\tau} e^{-\sigma M} \mathbf{a}\left(e^{\sigma M} \mathbf{y}\right) d \sigma-\tau \int_{0}^{1} e^{-\sigma M} \mathbf{a}\left(e^{\sigma M} \mathbf{y}\right) d \sigma\right]\right]\left(e^{-\tau M} \mathbf{a}\left(e^{\tau M} \mathbf{y}\right)\right)\right. \\
& \left.-\left[\nabla\left[\int_{0}^{1} e^{-\sigma M} \mathbf{a}\left(e^{\sigma M} \mathbf{y}\right) d \sigma\right]\right]\left(\int_{0}^{\tau} e^{-\sigma M} \mathbf{a}\left(e^{\sigma M} \mathbf{y}\right) d \sigma-\tau \int_{0}^{1} e^{-\sigma M} \mathbf{a}\left(e^{\sigma M} \mathbf{y}\right) d \sigma\right)\right) \\
& \quad+\int_{0}^{1}\left([\nabla \nabla V]\left(\int_{0}^{\tau} e^{-\sigma M} \mathbf{a}\left(e^{\sigma M} \mathbf{y}\right) d \sigma-\tau \int_{0}^{1} e^{-\sigma M} \mathbf{a}\left(e^{\sigma M} \mathbf{y}\right) d \sigma\right)\right) \\
& \quad\left(\left(e^{-\tau M} \mathbf{a}\left(e^{\tau M} \mathbf{y}\right)\right)-\int_{0}^{1} e^{-\sigma M} \mathbf{a}\left(e^{\sigma M} \mathbf{y}\right) d \sigma\right) d \tau
\end{aligned}
$$

The integrand in the last term is the dot product of a symmetric matrix (not depending on $\tau$ ) applied to a vector with the $\tau$-derivative of this same vector; so it is an exact $\tau$-derivative. Consequently, the last term is zero. Beside this, integrating by parts the first piece of the first term of the right 
hand side of 3.69 gives:

$$
\begin{aligned}
& \int_{0}^{1}\left(\left[\nabla\left[\int_{0}^{\tau} e^{-\sigma M} \mathbf{a}\left(e^{\sigma M} \mathbf{y}\right) d \sigma-\tau \int_{0}^{1} e^{-\sigma M} \mathbf{a}\left(e^{\sigma M} \mathbf{y}\right) d \sigma\right]\right]\left(e^{-\tau M} \mathbf{a}\left(e^{\tau M} \mathbf{y}\right)\right)\right. \\
& \left.-\left[\nabla\left[\int_{0}^{1} e^{-\sigma M} \mathbf{a}\left(e^{\sigma M} \mathbf{y}\right) d \sigma\right]\right]\left(\int_{0}^{\tau} e^{-\sigma M} \mathbf{a}\left(e^{\sigma M} \mathbf{y}\right) d \sigma-\tau \int_{0}^{1} e^{-\sigma M} \mathbf{a}\left(e^{\sigma M} \mathbf{y}\right) d \sigma\right)\right) . \\
& (\nabla V) d \tau= \\
& -\int_{0}^{1}\left(\left[\nabla\left[e^{-\tau M} \mathbf{a}\left(e^{\tau M} \mathbf{y}\right)-\int_{0}^{1} e^{-\sigma M} \mathbf{a}\left(e^{\sigma M} \mathbf{y}\right) d \sigma\right]\right]\left(\int_{0}^{\tau} e^{-\sigma M} \mathbf{a}\left(e^{\sigma M} \mathbf{y}\right)\right)\right. \\
& -\left[\nabla\left[\int_{0}^{1} e^{-\sigma M} \mathbf{a}\left(e^{\sigma M} \mathbf{y}\right) d \sigma\right]\right]\left(\int_{0}^{\tau} e^{-\sigma M} \mathbf{a}\left(e^{\sigma M} \mathbf{y}\right) d \sigma\right) \\
& \left.+\left[\nabla\left[\int_{0}^{1} e^{-\sigma M} \mathbf{a}\left(e^{\sigma M} \mathbf{y}\right) d \sigma\right]\right]\left(\tau \int_{0}^{1} e^{-\sigma M} \mathbf{a}\left(e^{\sigma M} \mathbf{y}\right) d \sigma\right)\right) \cdot(\nabla V) d \tau= \\
& \int_{0}^{1}\left(\left[\nabla\left[e^{-\tau M} \mathbf{a}\left(e^{\tau M} \mathbf{y}\right)\right]\right]\left(\int_{0}^{\tau} e^{-\sigma M} \mathbf{a}\left(e^{\sigma M} \mathbf{y}\right)\right)\right. \\
& \left.+\left[\nabla\left[\int_{0}^{1} e^{-\sigma M} \mathbf{a}\left(e^{\sigma M} \mathbf{y}\right) d \sigma\right]\right]\left(\tau \int_{0}^{1} e^{-\sigma M} \mathbf{a}\left(e^{\sigma M} \mathbf{y}\right) d \sigma\right)\right) \cdot(\nabla V) d \tau
\end{aligned}
$$

Using this and injecting in (3.64, allows us to claim the following Theorem.

Theorem 3.11. Under assumption of Lemma 3.9, function $V_{1}(t, \mathbf{y})$ linked by Lemma 3.10 with the Two-Scale limit $U_{1}(t, \tau, \mathbf{x})$ of $\left(u^{\varepsilon}-[U]^{\varepsilon}\right) / \varepsilon$ is solution to

$$
\begin{aligned}
& \frac{\partial V_{1}}{\partial t}+\left(\int_{0}^{1} e^{-\sigma M} \mathbf{a}\left(e^{\sigma M} \mathbf{y}\right) d \sigma\right) \cdot \nabla V_{1}= \\
& \quad\left(\int _ { 0 } ^ { 1 } \left(\left[\nabla\left[e^{-\tau M} \mathbf{a}\left(e^{\tau M} \mathbf{y}\right)\right]\right]\left(\int_{0}^{\tau} e^{-\sigma M} \mathbf{a}\left(e^{\sigma M} \mathbf{y}\right)\right) d \tau\right.\right. \\
& \left.\left.\quad+\frac{1}{2}\left[\nabla\left[\int_{0}^{1} e^{-\sigma M} \mathbf{a}\left(e^{\sigma M} \mathbf{y}\right) d \sigma\right]\right]\left(\int_{0}^{1} e^{-\sigma M} \mathbf{a}\left(e^{\sigma M} \mathbf{y}\right) d \sigma\right)\right)\right) . \\
& V_{1 \mid t=0}=0 .
\end{aligned}
$$

Remark 3.12. Uniqueness of the solution of (3.71) and (3.72) leads that the whole sequence $\left(u^{\varepsilon}-\right.$ $\left.[U]^{\varepsilon}\right) / \varepsilon$ converges.

\subsection{For the numerics}

In the case when $\varepsilon$ is small, computing a numerical approximation of 3.1 can be expensive in term of CPU time since it requires a time step which is small compared with $\varepsilon$.

If the result we just set out is reinterpreted, it can be claimed that

$$
u^{\varepsilon}(t, \mathbf{x}) \sim\left[V\left(t, e^{-\tau M} \mathbf{x}\right)+\varepsilon\left(V_{1}\left(t, e^{-\tau M} \mathbf{x}\right)+W_{1}\left(t, \tau, e^{-\tau M} \mathbf{x}\right)\right)\right]_{\mid \tau=t / \varepsilon},
$$

where $V$ is given as the solution of equation 3.23 and 3.24 which neither contain or generate small oscillations in its solution, $W_{1}$ is explicitly given in terms of $V$ by (3.53) and where $V_{1}$ is also solution of a problem without oscillation: 3.71 and 3.72.

This approach can be used to build numerical methods called Two-Scale Numerical Methods. Such an approach was used in Ailliot, Frénod \& Monbet [1], Frénod, Mouton \& Sonnendrücker [17], Frénod, Salvarani \& Sonnendrücker [19] and Mouton [25]. 


\section{REFERENCES}

[1] P. Ailliot, E. Frénod, and V. Monbet. Long term object drift forecast in the ocean with tide and wind. Multiscale Modeling and Simulations, 5(2):514-531, 2006.

[2] P. Ailliot, E. Frénod, and V. Monbet. Modeling the coastal ocean over a time period of several weeks. Journal of Differential Equations, 248:639-659, 2010.

[3] G. Allaire. Homogenization and Two-scale Convergence. SIAM Journal on Mathematical Analysis, 23(6):14821518,1992

[4] M. Amar. Two-scale convergence and homogenization on BV( $\omega)$. Asymptotic Analysis, 65(1):65-84, 1998.

[5] A. Bensoussan, J. L. Lions, and G. Papanicolaou. Asymptotic analysis for periodic structures. Studies in Mathematics and its Applications, Vol. 5. North Holland, 1978.

[6] S. Bochner. Integration von funktionen, deren werte die elemente eines vectorraumes sind. Fundamenta Mathematicae, 20:262-276, 1933.

[7] A. Braides. $\Gamma$-convergence for beginners. Oxford University Press, 2002.

[8] J. Casado-Díaz and I. Gayte. The two-scale convergence method applied to generalized Besicovitch spaces. Proceedings of the Royal Society of London. Series A: Mathematical, Physical and Engineering Sciences, 458(2028):2925-2946, 2002.

[9] G. Dal Maso. An introduction to Г-convergence. Birkhäuser, Basel, 1993.

[10] J. Diestel and J. J. Uhl. Vector measures. Transaction of the American Mathematical Society, 198:253-271, 1977.

[11] B. Engquist. Computation of oscillatory solutions to partial differential equations. In Claude Carasso, Denis Serre, and Pierre-Arnaud Raviart, editors, Nonlinear Hyperbolic Problems, volume 1270 of Lecture Notes in Mathematics, pages 10-22. Springer Berlin / Heidelberg, 1987. 10.1007/BFb0078314.

[12] I. Faye, E. Frénod, and D. Seck. Long term behaviour of singularly perturbed parabolic degenerated equation. Submitted.

[13] I. Faye, E. Frénod, and D. Seck. Singularly perturbed degenerated parabolic equations and application to seabed morphodynamics in tided environment. Discrete and Continuous Dynamical Systems - Serie A, 29(3):1001-1030, 2011.

[14] E. Frénod. Homogénéisation d'équations cinétiques avec potentiels oscillants. PhD thesis, Université Paris Nord, Av J. B. Clément, F-93400 Villetaneuse, 121994.

[15] E. Frénod. Application of the averaging method to the gyrokinetic plasma. Asymp. Anal., 46(1):1-28, 2006.

[16] E. Frénod and K. Hamdache. Homogenisation of kinetic equations with oscillating potentials. Proc. Royal Soc. Edinburgh, 126A:1247-1275, 1996.

[17] E. Frénod, A. Mouton, and E. Sonnendrücker. Two scale numerical simulation of the weakly compressible 1d isentropic Euler equations. Nümerishe Mathmatik, 108(2):263-293, 2007.

[18] E. Frénod, P. A. Raviart, and E. Sonnendrücker. Asymptotic expansion of the Vlasov equation in a large external magnetic field. J. Math. Pures et Appl., 80(8):815-843, 2001.

[19] E. Frénod, F. Salvarani, and E. Sonnendrücker. Long time simulation of a beam in a periodic focusing channel via a two-scale PIC-method. Mathematical Models and Methods in Applied Sciences, 19(2):175-197, 2009.

[20] E. Frénod and E. Sonnendrücker. Homogenization of the Vlasov equation and of the Vlasov-Poisson system with a strong external magnetic field. Asymp. Anal., 18(3,4):193-214, Dec. 1998.

[21] E. Frénod and E. Sonnendrücker. Long time behavior of the two dimensionnal Vlasov equation with a strong external magnetic field. Math. Models Methods Appl. Sci., 10(4):539-553, 2000.

[22] E. Frénod and E. Sonnendrücker. The Finite Larmor Radius Approximation. SIAM J. Math. Anal., 32(6):1227$1247,2001$.

[23] E. Frénod and F. Watbled. The Vlasov equation with strong magnetic field and oscillating electric field as a model for isotop resonant separation. Elec. J. Diff. Eq., 2002(6):1-20, 2002.

[24] P. Gérard. Microlocal defect measures. Communications in Partial Differential Equations, 16(11):1761-1794, jan 1991.

[25] A. Mouton. Two-scale semi-lagrangian simulation of a charged particles beam in a periodic focusing channel. Kinet. Relat. Models, 2(2):251-274, 2009.

[26] F. Murat. H-convergence. Séminaire d'Analyse Fonctionnelle et Numérique d'Alger, 1977.

[27] G. Nguetseng. A general convergence result for a functional related to the theory of homogenization. SIAM Journal on Mathematical Analysis, 20(3):608-623, 1989.

[28] G. Nguetseng. Asymptotic analysis for a stiff variational problem arising in mechanics. SIAM Journal on Mathematical Analysis, 21(6):1394-1414, 1990.

[29] G. Nguetseng and N. Svanstedt. $\Sigma$-convergence. Banach Journal of Mathematical Analysis, 5(1):101-135, 2011.

[30] G. Nguetseng and J.-L. Woukeng. $\Sigma$-Convergence of nonlinear parabolic operators. Nonlinear Analysis: Theory, Methods 86 Applications, 66(4):968-1004, feb 2007.

[31] H. E. Pak. Geometric two-scale convergence on forms and its applications to Maxwell's equations. Proceedings of the Royal Society of Edinburgh, 135A:133-147, 2005.

[32] A. A. Pankov. G-convergence and homogenization of nonlinear partial differential operators. Springer, sep 1997. 
[33] E. Sanchez-Palencia. Nonhomogeneous media and vibration theory, volume 127 of Lecture Notes in Physics. Springer-Verlag, Berlin, 1980.

[34] L. Schwartz. Analyse III : calcul intégral. Hermann, 1993.

[35] L. Tartar. Cours Peccot. Collège de France, 1977.

[36] L Tartar. Compensated compactness and applications to partial differential equations. In Nonlinear Analysis and Mechanics, Heriot-Watt Symposium 4, pages 136-211. 1979.

[37] L. Tartar. H-measures, a new approach for studying homogenisation, oscillations and concentration effects in partial differential equations. Proceedings of the Royal Society of Edinburgh: Section A Mathematics, 115(34):193-230, jan 1990.

[38] L. Tartar. The General Theory of Homogenization. A Personalized Introduction. Springer Verlag, dec 2009.

[39] K Yosida. Functional analysis. 6.ed. Grundlehren der Mathematischen Wissenschaften [Fundamental Principles of Mathematical Sciences], 123. Springer-Verlag, Berlin-New York, 1980. 